\title{
Imaging sensor data modelling and evaluation based on optical composite characteristics
}

\author{
Investigation of data quality for inline inspection
}

\author{
Sebastian Meister ${ }^{1}$ (D) . Lars Grundhöfer ${ }^{2} \cdot$ Jan Stüve $^{1} \cdot$ Roger M. Groves $^{3}$
}

Received: 29 December 2020 / Accepted: 24 June 2021 / Published online: 25 July 2021

(C) The Author(s) 2021

\begin{abstract}
Automated Fibre Placement is a common manufacturing technique for composite parts in the aero-space industry. Therefore, a visual part inspection is required which often covers up to $50 \%$ of the actual production time. Moreover, the inspection quality of this manual step fluctuates significantly. A camera-based automated inline inspection is capable of increasing the inspection efficiency and accuracy. However, the interpretability of the acquired data strongly depends on the sensor configuration and the inspected material. Thus, this paper introduces methods for modelling and assessing an imaging sensor on the example of a composite material reflecting a spot laser to a camera sensor. In this context, the reflection properties of the material are incorporated into a simulation and validated in comparison to real camera images from the experimental setup. The EMVA 1288 sensor model in combination with the Cramér-Rao lower bound indicates a feasible estimability of the beam propagation, but shows limitations in the predictability of the number of incident photons. The laser spot analysis indicated that the laser spot can deviate from an exact oval shape but its peak value is suitable for robust spot identification in an image. The outlined methodology is also adaptable to other imaging sensors, illumination sources and materials. Thus, the findings can be useful for other fields and manufacturing processes.
\end{abstract}

Keywords Automated fibre placement · Inline inspection · Laser line scan sensor · Image quality metrics · Cramér-Rao lower bound $\cdot$ Sensor modelling

\section{Introduction}

Lightweight composites are applied more frequently in modern aerospace production processes. The fuselage and wing manufacturing for the Boeing 787 as well as the Airbus A350 XWB are examples of a growing need for such lightweight components $[1,2]$. These parts are often made from Carbon Fibre-Reinforced Plastic (CFRP) due to its

Sebastian Meister

sebastian.meister@dlr.de

1 Center for Lightweight Production Technology, German Aerospace Center, Ottenbecker Damm 12, Stade, Germany

2 Institute of Communications and Navigation, German Aerospace Center, Kalkhorstweg 53, Neustrelitz, Germany

3 Aerospace Non-Destructive Testing Laboratory, Delft University of Technology, Kluyverweg 1, 2629 HS Delft, The Netherlands superior stiffness and strength properties in comparison to metallic components. In order to reduce the costs of the expensive manufacturing processes, efficient production techniques are of great interest. An appropriate automated fibre layup technology is one way to increase the production efficiency. Therefore, a subsequent visual inspection is mandatory to fulfil the high safety standards of the aerospace industry.

Nowadays this manual inspection needs between 32\% [3] and $50 \%$ [4] of the entire manufacturing time. Sometimes it is even impossible to ensure the required inspection precision due to the manual process. This provides considerable potential for optimisation in terms of quality and speed.

The first critical stage of an automated inline inspection system is a sufficiently accurate image data acquisition. A Laser Line Scan Sensor (LLSS) can be used for this purpose. Therefore, our previous studies from Meister et al. [5] already illustrated the major influence of the quality and characteristics of the input data for subsequent data analysis 
tasks. However, the data quality is influenced from different factors related to the sensor system and the viewed fibre material.

For this reason, in this paper we initially present a method for modelling and simulating the camera sensor for the observation of a spot laser. This procedure is based on a modified European Machine Vision Association (EMVA) 1288 modelling [6] and is inspired from the tutorial of Chao et al. [7]. This model serves to evaluate the sensor behaviour for various scenarios. Furthermore, the shape of a spot laser illumination reflected from a fibre composite is investigated in detail. Thus, we are able to derive optimisation strategies for the sensor parameters and the sensor setup. Moreover, appropriate Image Quality Assessment (IQA) methods are applied to evaluate the quality of the output image. Additionally, the sensor model and the determined material properties are linked within a simulation.

The Automated Fibre Placement (AFP) process is a quite novel technique for prepreg fibre material deposition which is increasingly used in industrial manufacturing [8-10]. In order to achieve a high transferability level of our findings, this approach provides the overall application case for this work. Hence a LLSS is often applied for the monitoring of AFP processes both in research and development, this sensor type is considered in this paper [5, 9, 11]. This sensor utilises the principle of laser camera triangulation to determine height information of laid up composite material from a projected laser beam. The research questions of this paper are:

I How should a mathematical model of a LLSS be designed to provide information on the quality of the resulting sensor image data for a specific application?

II Which techniques are suitable for evaluating the image quality of a spot laser reflected from a fibre composite material?

The methodology of this paper is to mathematically model and simulate the sensor in accordance with the EMVA 1288 standard. Based on this the Fisher Information and the Cramér-Rao lower bound are determined to closely investigate the sensor behaviour. Afterwards, the output image quality is assessed using the Peak Signal-to-Noise Ratio (PSNR) [12] and the speckle contrast [13] as well as the amplitude and width at standard deviation intensity level of a 2D Gaussian approximated imaged laser spot. Wang et al. [14] inspired us to analyse these parameters from a Gaussian laser spot fitting. Finally, the simulation results are validated in comparison to the real measurement images.

The results of this paper are particularly valuable for developers and operators of image-based inspection systems. Our results provide the basis for accelerated sensor parametrisation and robust data recording for the inspection of various fibre composites.

\section{Related research}

This section discusses the theoretical background for the use case considered. Furthermore the related research for modelling and validation purposes in this paper is outlined. Therefore, the application case and the applied sensor technology are presented.

Afterwards, methods for recording the laser spot and modelling the sensor and beam propagation properties are introduced.

\subsection{Manufacturing process}

In this section the fibre placement process is outlined. These days different fibre placement technologies are available. The AFP, Dry Fibre Placement (DFP), Automated Tape Laying (ATL) and Direct Roving Placement (DRP) are the most common from research and industrial production [15-17]. These techniques place fibre composite material in layers on a tool. The placed fibre material consists of unidirectional (UD) filaments. Prepreg composites embed these filaments within a polymer matrix. In contrast, for dry fibre materials they are usually superficially covered with a thermoplastic binder. Campbell [18] presents these techniques in details. Such a fibre placement procedure and the applied UD material are schematically illustrated in Fig. 1.

In particular the AFP technology is utilised to manufacture complex composite parts. Furthermore, it is suitable for manufacturing different components with varying geometries. This method is increasingly used in industrial aerospace manufacturing, but is still a quite novel process. However, Rudberg [19] indicates that the AFP technology will be installed more frequently in future manufacturing processes. Thus, we selected the AFP process for UD prepreg material to achieve a high transferability level of our findings [20]. In this process an effector places multiple narrow stripes of material (tows) at a velocity $\mathbf{v}$ along a given path (course). At the moment of placement a heating system heats the fibre material to achieve better tack properties. Then, the compacting roller presses the material onto the mould with the force $\mathbf{F}$ [15]. Accordingly, every part consists of several layers of fibre material [18].

During this production stage different defects might arise. These are usually directly related to the lay up process itself [20]. For this reason, Harik et al. [21] have examined the connection between an AFP defect and the corresponding process planning, layup strategies and machining. 
Fig. 1 AFP process with a heating system and a compaction roller to apply heat and pressure to the laid up UD fibre material. The prepreg material is made from parallel aligned filaments embedded in a polymer matrix. Here $\mathbf{v}$ represents the effector velocity and $\mathbf{F}$ the compaction force

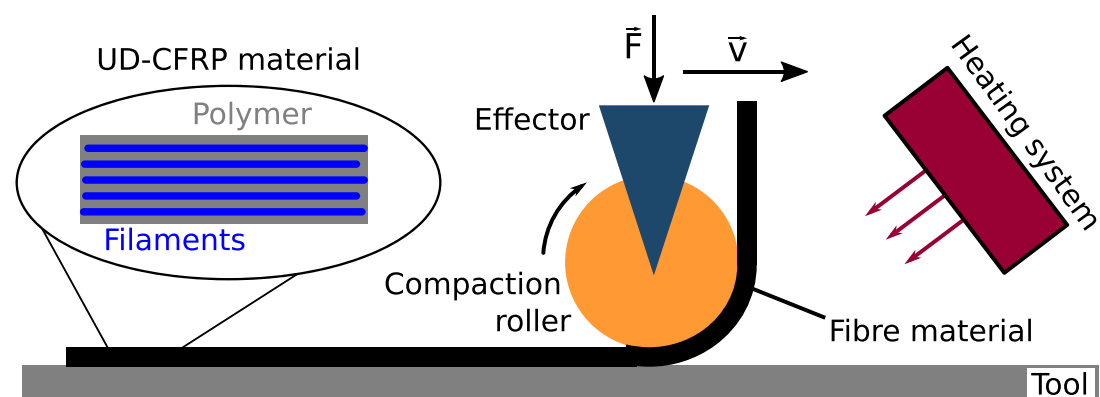

Additionally, Potter [22] has investigated effects which lead to AFP process deviations. They all stated, that the possible fibre layup defects always result in geometrical deviations from an accurate lay up surface. Therefore, it is beneficial to implement a system for automated inspection of these defects into the process. Corresponding sensor systems for data recording and inspection are presented in the following section.

\subsection{Data recording and inspection systems}

Process integrated monitoring for the AFP production is a frequently discussed subject in research and industry. Thus, several sensor principles for capturing sufficient inspection data have been studied. The Fraunhofer Institute for Integrated Circuits (IIS) investigated methods based on the polarisation effect [23, 24]. Moreover, the National Aeronautics and Space Administration (NASA) and the Institute of Production Engineering and Machine Tools - University Hanover (IFW) examined thermographic imaging inspection [25-27]. Both principles only enable the recording of two dimensional images.

Thus, InFactory Solutions [9], Profactor [28], Electroimpact $[8,10]$ and Danobat Composites [29] have build systems on the basis of the laser camera triangulation principle. Accordingly, they applied these LLSS devices for inline monitoring of laid up CFRP material in the AFP processes. One major advantage of the laser triangulation principle is the inherent calculation of topographical surface data. This might be the key to success of this measurement principle for the AFP inspection [9]. Accordingly, Sun et al. [30] summarised several inspection systems and their corresponding properties.

Cemenska et al. [8] reported that the total production time can be reduced by up to $20 \%$ through the use of automated camera-based inspection systems. Moreover they stated that, 90 to $99 \%$ of all manufacturing defects that occur can be detected and categorised. Black [10] summarised in her article that the application of such image-based inspection systems can even save between 30 and $60 \%$ of the production time. She also stated that course boundaries and tow endings are detected with about
92\% precision. In the those references they gave certain accuracy estimates. But in reality the comparison between the manual and automated inspection accuracy as well as the estimation of resulting improvements in accuracy are difficult, since the total number of defects in a component must be determined somehow.

The procedure for measuring fibre composite material with a LLSS is shown in Fig. 2.

For this technique a laser line is projected onto a surface and reflected back to a camera. The laser and the camera are aligned at an angle to each other. In order to measure the surface topology the entire laser-camera system is moved perpendicular to the laser line, in parallel to the surface. An image of each laser line height profile is captured with a certain time interval and a defined exposure time. Based on the laser line position within this image and the concatenation of several images, a surface depth map is estimated. Thus, Forest et al. [31] introduced the FIR filter for laser line detection within a sensor image. The company Automation Technology GmbH [32] implemented the FIR filter technique for their 3D LLSS sensors for a better laser peak detection from the acquired sensor image. This algorithm analyses the intensity distribution of the image with the aim to determine the laser line position in the sensor image. For this reason the zero crossing of the first derivative of the laser intensity image is estimated.

Moreover, Schmitt et al. [33, 34] examined LLSS techniques for the scanning of fabrics and preforms. They reached sub-pixel precision for the contour scanning. Thus, they prove that a LLSS is suitable for the CFRP inspection. Miesen et al. [35] investigated an inspection process using a point laser displacement system. Amir and Thörnberg [36] researched various image processing techniques with the objective of reducing disturbances within LLSS image data. For this purpose they examined the influences of noise, dynamics in light intensity, and surface scattering on the example of metallic surface inspection. They also deal explicitly with the influence of laser speckle on the precision of a measurement. Concludingly, they provide a detailed overview of the characteristics of a LLSS. In addition, the considered surface has a major influence on the quality of a measurement. Thus, the following section discusses ways 
Fig. 2 Principle of the LLSS data acquisition and calculation of a height profile measurement image of a Lambertian scattering fibre material surface using the Finite Impulse Response (FIR) filter. $\beta$ represents the irradiation angle

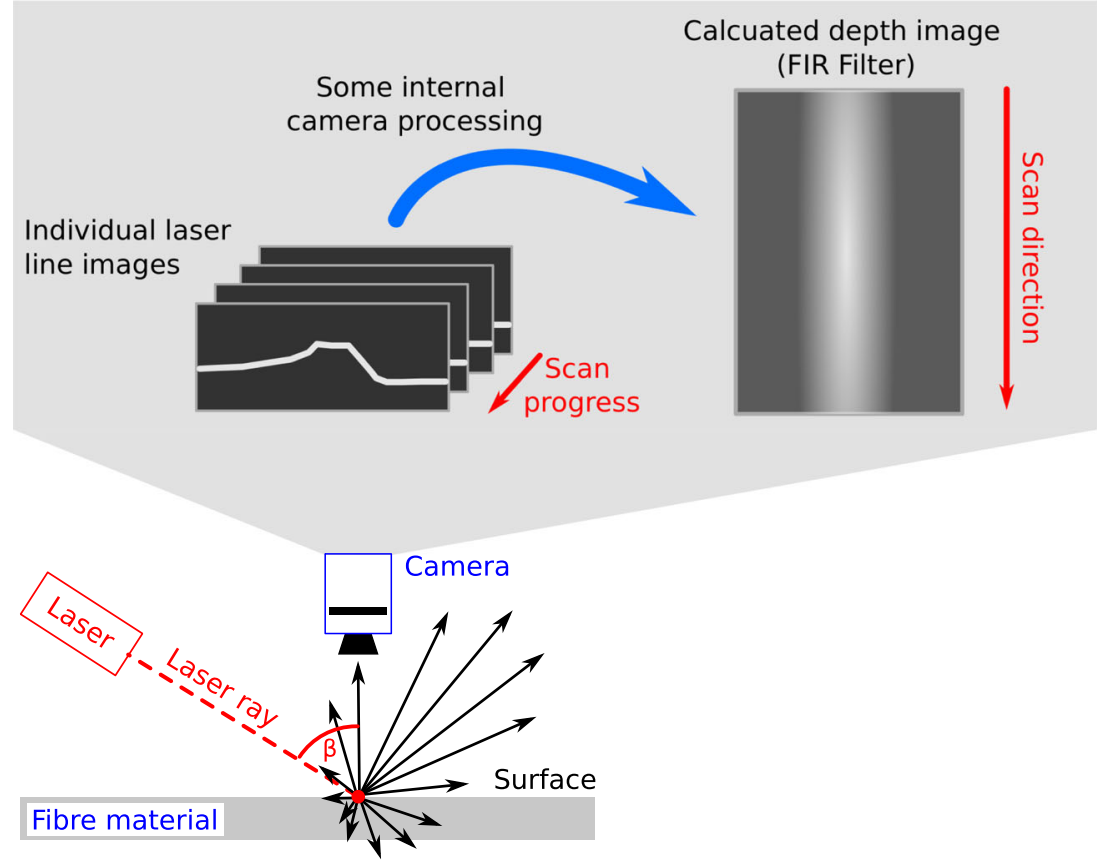

to characterise the optical properties of a fibre composite surface.

\subsubsection{Sensor and laser spot model}

Initially, a model is required which represents the camera sensor with the associated influencing parameters. For this purpose Jähne [37] and Rosenberger et al. [38] introduced the generic black-box camera model which is now incorporated into the EMVA 1288 standard [6]. In their research, they established a series of methods to analyse and visualise particular characteristics of imaging sensors. This model is schematically illustrated in Fig. 3.

For this modelling the magnification of the lens (M) from Eq. 1 is considered. This is approximately the ratio of the focal length (f) to the working distance (w). This also equals

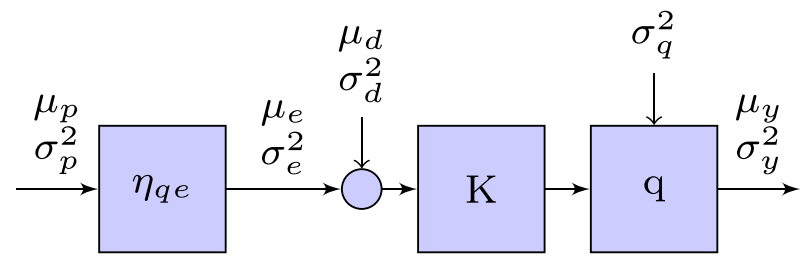

\section{\begin{tabular}{|l|l|}
\hline Photons & Digital value \\
\hline
\end{tabular}}

$\begin{array}{ccc}\text { Input: } & \text { Camera } & \text { Output } \\ \text { Irradiance } & \text { model } & \text { image }\end{array}$

Fig. 3 The diagram illustrates the camera model considered, which is inspired by the EMVA 1288 model [6] the quotient of the size of an image object $s_{i}$ to the real size of the corresponding object $s_{o}[39,40]$.

$M \approx \frac{f}{w} \approx \frac{s_{i}}{s_{o}}$

On this basis Kube [41] investigated photon transfer measurements based on Signal-to-Noise Ratio (SNR). Thus, he presents them for Charge Coupled Device (CCD) cameras, which were applied for various beam profile diagnostics at his institute. Furthermore, Jauregui-Sanchez et al. [42] investigated the performance and noise characteristics of individual photo detectors. Therefore, they applied a suitable sensor model which consists of various types of noise and other physical characteristics. This model represents the relationship between incident photons, the corresponding electrical charge and the resulting image. They examined the sensors SNR as a function of power and different properties of the incoming radiation.

In order to model a reflected laser spot within a camera image plane an appropriate laser propagation model is needed. For this purpose Wang et al. [14] presented a method for composite inspection using a spot laser and thermographic imaging. With the aim to model the inter material laser beam propagation, they applied a Finite Element Method (FEM)-based approach on the basis of Fast Fourier Transform (FFT) calculations. For the modelling of the inter material propagation of the laser, a Gaussian beam modelling with an expanded standard deviation of the beam profile is applied. Stokes-Griffin and Compston [43] give a more detailed explanation about the interaction of a laser with CFRP material. They investigated in their paper 
the optical properties of a thermoplastic CFRP for heating with a near infrared laser. For this purpose they examined distinct absorption properties of the material. Furthermore, they outlined the top hat shape of the installed laser.

Beyond that, Chao et al. [7] explained several approaches for modelling camera sensor data. They discussed different camera data models and their inherent characteristics. Furthermore they presented ways for calculating the Fisher Information Matrix (FIM) and the corresponding CramérRao lower bound (CRLB) exemplary for an imaging microscope application under the observation of a single molecules. They also consider ways of determining the required parameters and measurement variables.

\subsubsection{Evaluation of an imaged laser spot}

In order to evaluate an image, several IQA metrics are available in the literature. However, in case a reference image is available for evaluation, Samajdar and Quraishi [12] as well as Zhao et al. [44] gave an overview of different IQA methods and their properties.

In addition, considering a laser beam reflected from a fibre composite material, this leads to speckle patterns. In order to evaluate the characteristics of laser beam reflections from CFRP, Coyotl-Ocelotl et al. [13] described the speckle contrast $C_{s}$ in their work. This value is defined in Eq. 2 as the ratio of the standard deviation $\sigma_{I}$ of the image I and the mean value of the considered laser image region $\bar{I}$.

$C_{s}=\frac{\sigma_{I}}{\bar{I}}$

In the following section, the methodologies for modelling, measuring and validating the application case under consideration is described.

\section{Methods}

Within this section at first the measurement setups for the acquisition of material and sensor characteristics is discussed. Then, the methodology for the actual image acquisition and laser spot evaluation is explained. Afterwards, the beam propagation modelling is introduced. Concluding, an approach for characterising optical material properties is presented. Moreover, the subsequent sections describe the mathematical modelling of the LLSS and the corresponding simulation.

In order to provide a clear overview, the symbols introduced and utilised in this section are listed in Table 1 together with their corresponding brief description.

\subsection{Optical laser camera setup}

In this section, first the general measurement assembly is described. Subsequently a reasonable aperture f-number is selected. Afterwards, the influence of interference and diffraction effects is briefly evaluated.

\subsubsection{Measurement setup}

The setup illustrated in Fig. 4 was used to investigate the expansion of the laser spot on the materials surface. Moreover, it is utilised to parametrise and validate the simulation.

This assembly consists of a Picotronic DC650-1-3(8x25)C500 spot laser [45] and an Automation Technology $\mathrm{GmbH}$ (AT) C5-4090 camera [46, 47] with a AMS CMV12000 [48] sensor chip. This setup is looking almost perpendicularly at a Hexel HexPly 8552 [49] IM7 fibre [50] material sample at a working distance of $d_{w}=250 \mathrm{~mm}$ each. This material has a Cured Ply Thickness (CPT) of about $131 \mu \mathrm{m}$ and a filament diameter of about $5.2 \mu \mathrm{m}$, which is also the approximate slit width between two filaments. The volume fraction of this material is around $57.70 \%$. According to our own reflectance measurements with an Opsira gonio'2pi-bsdf custom Gonioreflectometer [51], the material has a relative degree of reflection of about $\varrho_{m} \approx 2 \%$ in the spectral range between $640 \mathrm{~nm}$ and $660 \mathrm{~nm}$ when observed perpendicularly. The acquired measurement images has a size of 4096 px (w) x 3072 px (h). The sensor image plane has the width of $s_{w}=22.528 \mathrm{~mm}$ and the height of $s_{h}=$ $16.896 \mathrm{~mm}$. Each pixel has a dimension of $s_{p x}=s_{p x, x}=$ $s_{p x, y}=5.5 \cdot 10^{-6} \mathrm{~m}$. Considering the manufacturers specifications for the size of a single pixel and the dimension of the image plane, the calculated fill factor is $100 \%$. Furthermore, the sensor specifications claim that micro-lenses are installed to increase the fill factor [48]. Thus, we assume that the non-light sensitive area between the individual pixels is negligible. The installed $P_{L}=1 \mathrm{~mW}$ laser has a nearly Gaussian beam profile with a wavelength of $\lambda_{l a}=650 \mathrm{~nm}$ and a coherence length of $l_{c}=1361 \mu \mathrm{m}$. In comparison, the STR-660 line laser from Coherent Inc. which is usually delivered with the AT C5-4090 LLSS, has a coherence length of a few millimetres $<<10 \mathrm{~mm}$ depending on the diode installed, the applied laser power and the ageing of the diode. Thus, the coherence lengths of both lasers are in a similar range of values. Moreover, the horizontal full width at half maximum (FWHM) beam width of the spot laser is $\mathrm{FWHM}_{l a, x}=72.8 \mu \mathrm{m}$ and in the vertical direction $\mathrm{FWHM}_{l a, y}=80.4 \mu \mathrm{m}$ [45]. The AT C5-4090 camera uses a Schneider Kreuznach Xenoplan 2.8/50-0902 lens [52] with focal length $f=50.2 \mathrm{~mm}$. Furthermore it has a built-in Midwest Optical Systems - BP660 dark red 
Table 1 List of symbols including a brief description, which are introduced in this section

\begin{tabular}{|c|c|}
\hline Symbol & Description \\
\hline$\alpha, \beta$ & Detection angle, illumination angle \\
\hline$\gamma$ & Sample/ fibre orientation \\
\hline$s_{i}$ & Sensor dimensions in i-th direction \\
\hline$s_{p x, i}$ & Pixel size i-th direction \\
\hline$P_{L}$ & Laser power \\
\hline$\lambda_{l a}$ & Laser wavelength \\
\hline$l_{c}$ & Coherence length \\
\hline $\mathrm{FWHM}_{l a, i}$ & Laser profile FWHM in i-th direction \\
\hline$\varrho_{f}$ & Degree of transmission (filter) \\
\hline$\varrho_{m}$ & Degree of reflection (composite) \\
\hline$d_{f}$ & Width between two filaments \\
\hline$d_{w}$ & working distance \\
\hline f & focal length \\
\hline$s_{O}$ & Actual size of observed object \\
\hline$s_{i}$ & Size of object on image plane \\
\hline $\mathrm{FOV}_{i}$ & Field of view in i-th direction \\
\hline M & Magnification optical setup \\
\hline$\delta_{i}$ & Viewing angle in i-th direction \\
\hline$E_{l a, \sigma}$ & Emitted laser irradiance at working distance over area at standard deviation position \\
\hline$E_{r m, l a}$ & Laser irradiance reflected from material \\
\hline$E_{p x}$ & Incident irradiance on the pixel $(i, j)$ \\
\hline $\bar{d}_{s}$ & Average speckle grain dimension \\
\hline DOF & Depth of focus \\
\hline$f_{a}$ & Aperture f-number \\
\hline $\mathrm{u}$ & Maximum acceptable blur size \\
\hline$\sigma_{g}$ & Reflected laser spot diameter on composites surface at standard deviation position \\
\hline$\sigma_{l a}$ & Laser intensity profile diameter at standard deviation position \\
\hline$p_{b, m}$ & Beam propagation parameter composite \\
\hline$p_{1}$ & Beam propagation factor from experiments \\
\hline$p_{2}$ & Beam propagation offset from experiments \\
\hline$t_{\text {exp }}$ & Exposure time camera \\
\hline$C_{s}$ & Speckle contrast \\
\hline$n_{s}$ & Number of speckle regions \\
\hline
\end{tabular}

bandpass filter [53]. For the considered laser wavelength $\lambda_{\text {la }}$, this BR660 filter has a transmission of $91.93 \%$ which means $\varrho_{f}=0.9193$ for the considered wavelength. Figure 5 illustrates the magnification and illumination relationships between the laser spot image on the sample and its representation in the camera image as well as the associated optical parameters.

According to the correlations from Section 2.2.1, a magnification factor of $M \approx \frac{f}{d_{w}} \approx 0.2$ can be determined for the considered assembly. Furthermore, considering the sensor dimensions, the Field of View (FOV) in the height direction is $\mathrm{FOV}_{h}=\frac{s_{h}}{f} d_{w} \approx 84.14 \mathrm{~mm}$ and in the width direction $\mathrm{FOV}_{w}=\frac{s_{w}}{f} d_{w}=112.19 \mathrm{~mm}$. This leads to a viewing angle in height direction of $\delta_{h}=2 \tan ^{-1}\left(\frac{\mathrm{FOV}_{h}}{2 d_{w}}\right) \approx$ $19.1^{\circ}$ and in width direction of $\delta_{w} \approx 25.29^{\circ}$. Due to the Gaussian laser profile we assumed that the maximum irradiation is at the laser spots centre point. This specifies the origin of the corresponding coordinate systems on the sample $E_{r m, l a}(x=0, y=0)$ and in the image $E_{p x}(i=$ $0, j=0)$. In this case no sub-pixel accuracy is considered. If two neighbouring pixels have the same global maximum value, the pixel located more to the left and more to the top of the image is selected as the origin.

\subsubsection{Selection of aperture size}

Moreover, the lens aperture should be set as small as possible to compensate for the negative depth of focus (DOF) effects. However, it should be noted that, depending 


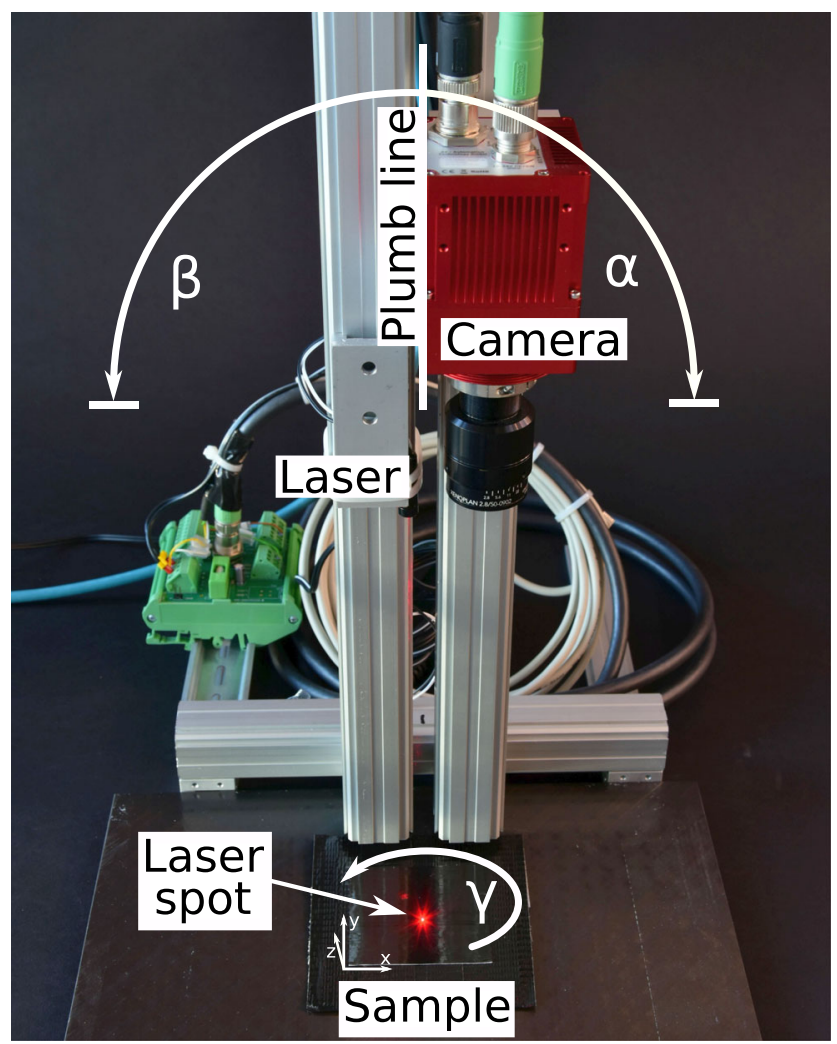

Fig. 4 Laser camera setup for tests. An Automation Technology GmbH C5-4090 camera and a Picotronic DC650-1-3(8x25)-C500 laser are utilised. For tests, the camera and laser are aligned almost perpendicular to the sample's surface. $\alpha \approx 0.5^{\circ}$ is the angle between plumb line and camera. $\beta \approx 0.5^{\circ}$ is the angle between plumb line and laser. $\gamma$ represents the sample rotation

on the application, a sufficient amount of light must illuminate the sensor. In addition, a very small aperture can cause increased diffraction and speckle effects in the camera image. The average speckle grain dimension $\bar{d}_{s}$ can be approximated from Eq. 3 [54] which was applied by Thompson et al. [55] and used from Tutsch et al. [56].

$\bar{d}_{s}=1.2(1+M) \lambda_{l a} f_{a}$

Moreover, the DOF can be approximated from Eq. 4 [57] as Tutsch et al. [56] explained.

$D O F \approx u f_{a} \frac{1+M}{M^{2}}$

According to Eq. 3 a $\bar{d}_{s}=0.936 f_{a} \mu \mathrm{m}$ is calculated from the given values. Moreover, the speckle contrast $C_{s}$ drops according to Eq. 5 with the square root of the number of speckle region $n_{s}$ [54].

$C_{s}=\frac{1}{\sqrt{n_{s}}}$

Due to this behaviour and following Thompson et al. [55] and Tutsch et al. [56] speckle can be reduced substantially when the average speckle size $\bar{d}_{s}$ is smaller than the dimension of a pixel $s_{p x}$ which means $\bar{d}_{s}<s_{p x}$. For the pixel size of the installed sensor, this implies a desired aperture number of $f_{a}<5.876$. However, for the considered application case, a comparatively large DOF from Eq. 4 is required. Inspired from Tutsch et al. [56] the maximal blur tolerance $\mathrm{u}$ should be one pixel. In order to provide suitable information even for more geometrically complex components and larger fibre placement defects, this should be at least between DOF $=[1.5,2] \mathrm{mm}$. Thus, the aperture f-number is sensibly in the interval $f_{a}=[9.1,12.1]$. For these reasons, an aperture of $f_{a}=11$ was chosen for all experiments in this paper. Due to Eq. 3 this leads to an average speckle size of $\bar{d}_{s}=10.296 \mu \mathrm{m}$. Thus, this equals 1.872 times the pixel dimension $s_{p x}$. In comparison to the case $\bar{d}_{s}=s_{p x}$ mentioned above, the speckle contrast in this scenario is increased by about $37 \%$.

\subsubsection{Diffraction and interference effects}

Additionally, due to the given coherence length and the Fraunhofer diffraction condition it is very likely that interference and diffraction effects occur for this setup. For this purpose, we assume that close together fibres of the material re-emit parts of the incident laser light. Thus, in this way small phase shifts can occur which can cause these interference effects. The coherence length of $l_{c}=$ $1361 \mu \mathrm{m}$ is quite large compared to the slit width of the fibre material of about $5.2 \mu \mathrm{m}$. Moreover, the Fraunhofer diffraction condition is valid:

$\frac{d_{f}^{2}}{d_{w} \lambda_{l a}}=\frac{\left(5.2 \cdot 10^{-6}\right)^{2} m^{2}}{0.25 \cdot 650 \cdot 10^{-9} m^{2}}=1.664 \cdot 10^{-4} \ll 1$

For this, $d_{f}=5.2 \mu \mathrm{m}$ is the width between two filaments and $d_{w}=250 \mathrm{~mm}$ is the working distance. However, due to the research from Wang et al. [14] as well as Stokes-Griffin and Compston [43] previously outlined in Section 2.2.1 the influences of interference and diffraction for this application case should be marginal. Thus, for the investigations in this paper we assume that the major influence on the variation in spot diameter and the variance in spot intensity result from the beam propagation within the material. For the subsequent modelling, only the global intensity maximum was considered. High order local maxima have lower intensities. Therefore, we did not examined these optical effects in detail. Nevertheless, they have to be taken into account when evaluating the results.

\subsection{Image acquisition and laser spot evaluation}

This section outlines the procedure for capturing suitable laser spot images. Furthermore, the applied evaluation methodology for these images is explained. 


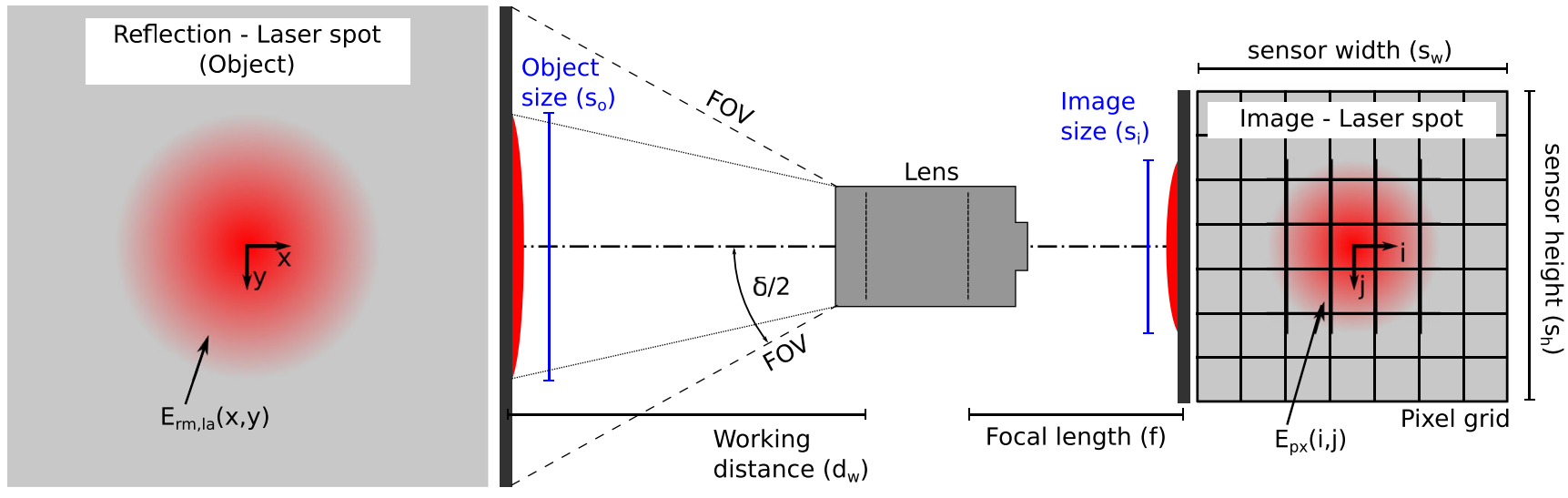

Fig. 5 Sketch of the optical setup and the different images of the considered laser spot. The working distance $d_{w}$, focal length f, object size $s_{o}$, image size $s_{i}$ and the half viewing angle $\delta / 2$ are displayed. Furthermore, the Field of View (FOV) is illustrated

\subsubsection{Image acquisition}

These methods are used to parametrise and validate the sensor model and the calculations in this paper. For this purpose, the test setup previously described in Section 3.1 was used. In order to investigate the speckle characteristics of the laser spot on the sample, the camera and laser were aligned almost perpendicular to the sample. Thus, $\alpha=$ $\beta \approx 0.5^{\circ}$, since the limitations from the sensor geometry prevent a smaller angle. Therefore, we assumed that the beam profile of the laser which is projected on the material is approximately as specified from the manufacturer for an accurate perpendicular view at a distance of $250 \mathrm{~mm}$. For detailed analysis of the speckle properties of the CFRP sample, 32 images were taken for $100 \mu s, 500 \mu s$ and $900 \mu s$ exposure time each. These exposure times were selected because the shortest integration time still allows sufficient light to reach the sensor in order to receive a measurable signal. On the other hand, the longest integration time comes close to the saturation level of the sensor, but without overexposure. To record these images, the sample orientation $\gamma$ was incrementally rotated by $45^{\circ}$ around its normal axis. Altogether, images were acquired for two entire cycles. For each rotation angle $\gamma$, the three images for the different exposure times were captured for exactly the same measuring points. Afterwards, the sample was further rotated.

\subsubsection{Evaluation of imaged reflected laser spot}

In order to improve processability, an image of size 240 x 240 px was cropped around the intensity maximum of the overall recorded image. In this case we assume that the centre of the reflected laser spot is roughly the intensity maximum of the measurement image. In order to measure intensity fluctuations in the image of the reflected laser spot on the sample, the previously specified speckle contrast was calculated for each entire reflection measurement image.

Regarding Sheikh et al. [58] as well as Samajdar and Quraishi [12], PSNR is particularly well suited to characterise white noise. Considering laser speckle patterns within an image as such a kind of white, granular image noise, the PSNR technique provides a very straightforward way to characterise a laser spot image with respect to the laser speckle. Accordingly, the PSNR values were calculated for each original image with the corresponding simulated image as reference. Additionally, the PSNR calculation indicates the degree of correspondence between two images. Typical values for 8-bit images are around 30$50 \mathrm{~dB}$ [59]. For 16-bit images, typical values are between 60-80 dB [60]. The sensor examined in this paper provides 16-bit images [47, 61], but due to the built-in sensor chip [48], only a 10-bit range of values is registered. For this reason, we expect a reasonable PSNR value for an acceptable match of the simulated and real captured laser spot images between the values for 8-bit and 16-bit images previously given. Thus, these PSNR value should be in the range of 50-60 $\mathrm{dB}$ for an adequate match of measurement and simulation.

Furthermore, a Gaussian fitting was applied to the laser spot intensity distribution of the measurement image. In this way the directional beam widths at standard deviation position of the reflected laser spot on the material sample were determined. All previously mentioned results were averaged over images of equal fibre orientations and displayed for the respective samples rotation angles $\gamma$. The corresponding measurement values were calculated for the physical and the simulated measurement images of the reflected laser spot. Thus, the aim was the validation of the simulation and the discussion of corresponding limitations. Below, an appropriate estimation for the laser spot beam propagation is explained. 


\subsection{Beam propagation modelling}

As indicated above, the emitted laser beam profile is Gaussian distributed with a certain spot size and the maximum irradiance at its centre. Furthermore, we know that the inter material beam propagation of the laser leads to an expansion of the reflected laser spot size. For investigations in this paper we assume the relationship from Eq. 7 for the overall beam diameter of the reflected laser spot at standard deviation position is $\sigma_{g}$. This is composed from a beam propagation parameter $p_{b, m} \in \mathbb{R}^{+}$and the diameter at standard deviation position of the laser spot $\sigma_{l a} \in \mathbb{R}^{+}$.

$\sigma_{g}=\frac{1}{M} p_{b, m}+\sigma_{l a}$

Due to the rather rough positioning of the laser spot and the material sample as well as the high variation in spot size due to the material properties, a direction independent average value for $\sigma_{g}$ was used in this paper.

In accordance with the literature from Section 2.2.1 like Eq. 8 we assume that the beam propagation factor $p_{b, m}$ increases with natural logarithm with the amount of incident photons. This is composed from the product of the laser irradiance at its standard deviation position $E_{l a, \sigma}$ and the exposure time $t_{\text {exp }}$.

$$
\begin{aligned}
& p_{b, m}\left(t_{\text {exp }}, E_{l a, \sigma}, p_{1}, p_{2}\right) \\
& =p_{1} \cdot \ln \left(E_{l a, \sigma} \cdot t_{\text {exp }}\right)+p_{2}
\end{aligned}
$$

Due to the fixed laser voltage and laser focus, $E_{l a, \sigma}=$ const. for the considered test case in this paper. Furthermore, the parameters $p_{1}$ and $p_{2}$ are material dependent parameters which influence the beam propagation inside the material.

These parameters need to be estimated experimentally. For this reason the natural logarithm of the three considered exposure times $\ln \left(t_{\text {exp }}\right)$ were linked to the previously estimated 2D Gaussian beam width at standard deviation intensity level. This fitting yields an approximated value for $p_{1}$ and $p_{2}$ for each rotation angle $\gamma$. Then the mean and standard deviation for $p_{1}$ and $p_{2}$ for each rotation angle $\gamma$ were calculated. For the investigations in this paper, the overall average value for $p_{1}$ and $p_{2}$ over all rotation angles $\gamma$ was then calculated and applied. The mentioned fitting was carried out in this paper with the polyfit function from the Numpy 1.19.0 library. Subsequently the measurement of further optical material properties is discussed.

\section{Sensor data modelling and evaluation}

The objective of this section is to build a pragmatic, mathematical sensor model for observing a laser spot in order to be able to carry out statistical analyses. In the following Section 5, this model is transferred to a simulation and some of the simplified assumptions, which are necessary for this theoretical evaluation, are adjusted for an improved simulation result.

At the beginning of this section the calculations for the modelling of the considered camera sensor are presented in detail. Subsequently, the FIM and CRLB calculations are discussed. The symbols introduced in this section as an extension to Table 1 are listed in Table 2 with their associated brief description.

Regarding the literature from Section 2.2.1 the EMVA 1288 standard provides an appropriate way to model a camera sensor. This method offers the possibility to easily transfer the approaches from this paper to other camerasensor systems for the inspection in lightweight composite manufacturing. Therefore, this is chosen for modelling in this paper. However, the original EMVA 1288 standard considers a homogeneous irradiation of the entire sensor. Since a point laser with a Gaussian beam profile was applied for the investigations in this study, the model was modified appropriately. In this modified version the mean value of several intensity maxima or alternatively the total energy of the laser from multiple sensor images characterise the sensor image plane.

This mathematical sensor model is then used to calculate the FIM and on this basis the CRLB. For this purpose, the photon distribution $f_{\theta}$ of a given emitting object $\mathrm{q}$ on the sensor image plane is modelled. On this basis the random variable $H_{\theta, k}$ specifies the number of detected electrons for each k-th pixel including noise. The sensor manufacturer provides the corresponding noise properties for our assembly [48]. The read out noise $\mu_{q}$ is not considered here due to its very small value compared to the laser peak signal. At the end the CRLB is exemplary evaluated for the number of incident photons and the laser spot dimension.

\subsection{Modelling sensor properties using EMVA 1288 standard}

This section models the AT C5-4090 camera sensor used in this paper. For this purpose, initially a single pixel is modelled and subsequently on this basis the entire image plane is described.

\subsubsection{Investigation of a single pixel}

Initially, we model the photons incident on a single pixel. This modelling is based on the intensity maximum of the image. Therefore, we assume that this maximum is located at the centre of the Gaussian distributed laser spots. Equation 9 gives the laser spot irradiance $E_{l a, \text { Gaus }}\left(r, z_{m}\right)$ 
Table 2 List of symbols including a brief description, which are additionally to Table 1 introduced in this section

\begin{tabular}{|c|c|}
\hline Symbol & Description \\
\hline$C_{k}$ & Sensor region \\
\hline$A_{p x}$ & Pixel area: $A_{p x}=s_{p x, x} \cdot s_{p x, y}$ \\
\hline$A_{\text {spot }}$ & Area of projected laser spot on surface \\
\hline$\varrho_{a}$ & Irradiance reduction from aperture: $\varrho_{a}=1 / f_{a}^{2}$ \\
\hline$\varrho_{s}$ & Influence factor - materials scattering \\
\hline$\theta$ & Parameter vector \\
\hline$\omega$ & Data \\
\hline$f_{\theta}$ & Photon distribution on sensor image plane \\
\hline$H_{\theta, k}$ & Amount of detected electrons in the k-th pixel \\
\hline$S_{\theta, k}$ & Independent Poisson random variable — \# photons converted into electrons \\
\hline$\mu_{\theta, k}$ & Corresponding mean value to $S_{\theta, k}$ \\
\hline$B_{\theta, k}$ & Independent Poisson random variable — \# detected electrons without irradiation \\
\hline$\beta_{\theta, k}$ & Corresponding mean value to $B_{\theta, k}$ \\
\hline$W_{\theta, k}$ & Gaussian random variable — \# electrons from readout noise \\
\hline$v_{\theta, k}$ & Mean number electrons — sensor signal \\
\hline$w\left(z_{m}\right)$ & Radius of Gaussian laser beam at a distance $z_{m}$ from the spot maximum \\
\hline $\mathrm{r}$ & Distance to propagation axis \\
\hline$\eta_{q e}$ & Quantum efficiency of sensor \\
\hline$\mu_{p}(i, j)$ & Mean number of photons at pixel $(i, j)$ \\
\hline$\mu_{p, t o t a l}$ & Mean total amount of photons on the sensor \\
\hline$\mu_{e}$ & Mean number of electrons \\
\hline$\mu_{y}(i, j)$ & Mean output signal at pixel $(i, j)$ \\
\hline$\mu_{y, \max }$ & Maximum mean value - output signal \\
\hline$\mu_{y, \text { total }}$ & Integral over output image \\
\hline$\mu_{y . d a r k}$ & Amplified mean dark noise \\
\hline$\mu_{d}$ & Mean dark noise \\
\hline$\mu_{q}$ & Mean readout noise \\
\hline$\sigma_{q}^{2}$ & Variance readout noise \\
\hline$E_{l a}(x, y)$ & Laser irradiance at $(\mathrm{x}, \mathrm{y})$ \\
\hline$E_{l a, \text { Gaus }}$ & Laser spot irradiance at certain Gaussian beam profile position \\
\hline$E_{l a, \max }$ & Maximum laser spot irradiance \\
\hline h & Planck constant \\
\hline $\mathrm{c}$ & Speed of light \\
\hline$y_{k}$ & k-th output image \\
\hline$q(x, y)$ & Point spread function at $(\mathrm{x}, \mathrm{y})$ \\
\hline $\mathrm{K}$ & Gain \\
\hline$\varrho$ & Correlation coefficient \\
\hline $\mathrm{z}$ & Photon counter \\
\hline$\rho_{\theta, k}$ & Probability Density Function for accumulated signal \\
\hline I & Fisher Information Matrix \\
\hline
\end{tabular}

over the Gaussian radiation profile with the standard deviation $\sigma_{l a}$ from Eq. 10. Then in Eq. 11 the maximum irradiance $E_{l a \text {,max }}$ is calculated at $\mathrm{r}=0$. In these equations, $P_{L}$ represents the laser power and $w\left(z_{m}\right)$ is the beam radius at a certain distance $z_{m}$ from the spot maximum at $z_{m}=0$.

$E_{l a, \text { Gaus }}\left(r, z_{m}\right)=\frac{2 P_{L}}{\pi w\left(z_{m}\right)^{2}} \exp \left(-2 \frac{r^{2}}{w\left(z_{m}\right)^{2}}\right)$

$$
\begin{aligned}
& \sigma_{l a}=\frac{F W H M}{2 \sqrt{2 \ln (2)}} \\
& \begin{aligned}
E_{l a, \text { max }} & =E_{l a, \text { Gaus }}\left(r=0, w=w_{\mathrm{FWHM}}\right) \\
& =\frac{2 P_{L}}{\pi w\left(z_{m}\right)^{2}}, \text { with } \\
w_{\mathrm{FWHM}} & =\frac{1}{2} \sqrt{\frac{2}{\ln (2)}} \mathrm{FWHM}
\end{aligned}
\end{aligned}
$$


The FWHM in this case defines the width of the Gaussian laser profile at the position where the intensity of the maximum is reduced by half. Therefore, the average of $\mathrm{FWHM}_{l a, x}$ and $\mathrm{FWHM}_{l a, y}$ from the laser manufacturers specifications from Section 3.1 is considered for the calculations in this paper. According to the EMVA 1288 model the number of incoming photons $\mu_{p}$ for each pixel $(\mathrm{i}, \mathrm{j})$ is calculated:

$$
\begin{aligned}
\mu_{p}(i, j) & =\frac{A_{p x} E_{p x}(i, j) t_{\text {exp }} \lambda_{l a}}{h c}, \text { with } \\
E_{p x}(i, j) & =E_{l a}(x, y) \varrho_{m} \varrho_{s} \varrho_{a} \varrho_{f}
\end{aligned}
$$

$A_{p x}$ represents the detecting area of a single pixel, $t_{\text {exp }}$ specifies the exposure time, $\mathrm{h}$ is the Planck constant and $\mathrm{c}$ is the speed of light. $E_{p x}(i, j)$ represents the irradiance for each pixel $(\mathrm{i}, \mathrm{j})$ on the sensor.

Since in this application a filter and a lens are installed in front of the sensor chip, appropriate reduction factors for the incoming irradiance must be taken into account. $\varrho_{a}$ describes the reduction in irradiance resulting from the aperture setting. This is $\varrho_{a}=\frac{1}{f_{a}^{2}}$, where $f_{a}$ represents the aperture f-number. $\varrho_{f}\left(\lambda_{l a}\right)$ is the transmission factor of the built-in filter for the given laser wavelength $\lambda_{l a}$. The parameters $f_{a}$ and $\varrho_{f}$ are introduced in Section 3.1. Additionally, the laser irradiance is significantly reduced due to the degree of reflection $\varrho_{m}$ of the fibre material viewed in this application.

Furthermore, $p_{b, m}$ represents the beam propagation factor from Eq. 8 previously discussed in Section 3.3. Accordingly, $\varrho_{s}\left(t_{\text {exp }}, p_{b, m}\right)$ from Eq. 13 normalises the number of incoming photons based on the enlarged laser spot area. For this the beam propagation factor $p_{b, m}$ and the exposure time $t_{\text {exp }}$ are considered. Therefore, it must be stated that the area of the laser sport $A_{\text {spot }} \sim \sigma_{l a}^{2}$. Additionally, $\mu_{p} \sim t_{\text {exp }}$ applies. For clarification, $\varrho_{s}$ is only a mathematical auxiliary factor to enable modelling in the given way.

$\varrho_{s}\left(t_{\text {exp }}, p_{1}, p_{2}\right)=\frac{1}{t_{\text {exp }}} p_{b, m}^{2}\left(t_{\text {exp }}, p_{1}, p_{2}\right)$

In order to calculate the total amount of photons on the sensor $\mu_{p, \text { total }}$ from Eq. 14, the number of detected photons $\mu_{p}(i, j)$ for each pixel needs to be integrated over its entire sensor area $C_{k}$.

$\int_{C_{k}} \mu_{p}=\mu_{p, t o t a l}=\frac{P_{L} t_{\text {exp }} \lambda_{l a}}{h c} \varrho_{m} \varrho_{s} \varrho_{a} \varrho_{f}$

According to the EMVA 1288 model a quantum efficiency parameter $\eta_{q e}$ is introduced in Eq. 15 to convert the number of incident photons into electrons generated on the sensor. $\eta_{q e}$ is provided from the data sheet of the sensor.

$\eta_{q e}=\frac{\mu_{e}}{\mu_{p}}$
According to the EMVA 1288 model from Fig. 3, the overall output signal of each pixel is calculated in Eq. 16 including the corresponding noise and gain influences.

$$
\begin{aligned}
\mu_{y}(i, j) & =\mu_{y . d a r k}+K \eta_{q e} \mu_{p}(i, j) \\
& =K \mu_{d}+K \eta_{q e} \frac{\lambda}{h c} A_{p x} E_{p x}(i, j) t_{e x p}
\end{aligned}
$$

Within this equation, $\mathrm{K}$ is the gain of the sensor. Furthermore, $\mu_{d}$ is the mean number of electrons counted without irradiation. This noise component $\mu_{d}(T)$ depends on the sensor temperature $\mathrm{T}$. The sensor chip manufacturer [48] provides corresponding values for $\mu_{d}(T)$. The EMVA 1288 standard specifies a linear relationship between the noise $\mu_{d}$ and the exposure time $t_{\text {exp }}$ for a constant sensor temperature $\mathrm{T}$ which leads to $\Delta \mu_{d}(T=$ const.$) \sim t_{\text {exp }}$. Accordingly, $\mu_{y . d a r k}=K \mu_{d}$ gives the dark noise. This is basically the mean image signal without irradiation. In accordance with the EMVA 1288 standard, the mean value of the readout noise $\mu_{q}$ is located in the middle of the quantisation interval. The variance of the corresponding readout noise can be approximated according to the EMVA 1288 model. This leads to $\sigma_{q}^{2}=\frac{1}{12} \mathrm{DN}^{2}$. The following section explains the procedure for applying the observations of a single pixel to the entire sensor.

\subsubsection{Image plane modelling}

This section describes the modelling of the entire sensor image plane. Within the EMVA 1288 standard a homogeneous irradiation of the sensor is required. On this basis the mean value of two images $\mathrm{K}=1$ is determined in Eq. 17 to characterise the sensor image plane. In this equation $\mathrm{K}+1$ images with $\mathrm{M}$ rows and $\mathrm{N}$ columns each are considered.

$\mu_{y}=\frac{1}{K M N+1} \sum_{k=0}^{K} \sum_{i=0}^{M} \sum_{j=0}^{N} y_{k}(i, j)$

However, since we are examining a laser spot with varying size in this application, this average value would change with the spot size or the dimensions of the sensor image. Therefore, the image average value is not meaningful here. For this reason, the modelling is slightly adjusted. In relation to Eq. 18 for this paper we average the maximum pixel intensity of the sensor over several input images.

$\mu_{y, \max }=\frac{1}{K+1} \sum_{i=0}^{K} \max \left(y_{k}\right) \approx \frac{1}{K+1} \sum_{k=0}^{K} y_{k}(0,0)$

This value $\mu_{y, \max }$ is independent of the image dimension or laser spot size and can even be transferred to other intensity distributions. However, it should be noted that this modelling is only applicable if there is one global intensity maximum which can be unambiguously assigned to a single pixel. 
For a more detailed subpixel analysis, a peak between pixels can also be used. However, for this modelling it is important, as described before, that there is only one global maximum per region of interest. However, several observation areas in one measurement image are also conceivable. But this is not part of the modelling in this study.

Moreover, such maximum value can also be utilised if the laser beam hits the surface at a different angle. In this case, it is noteworthy that the size of the laser spot changes with different angles, and thus, the total power of the laser is distributed over another area. Accordingly the maximum value mentioned above changes, but can still be applied as a criterion. As already explained above and illustrated in Fig. 5, we define the maximum irradiance of the measurement image as the origin of the Gaussian intensity distribution of the point laser. For this reason we can state that the maximum of the i-th output image $\max \left(y_{i}\right)$ is the image centre $y_{i}(0,0)$. This corresponds to the origin of the laser profile. Thus, $\max \left(y_{i}\right) \approx y_{i}(0,0)$ applies.

In addition, following the idea of Eq. 14, the inhomogeneous image of the reflected laser spot can be characterised as the sum of all individual pixel intensity values of the camera image by:

$\mu_{y, \text { total }}=\frac{1}{K+1} \sum_{k=0}^{K} \sum_{i=0}^{M} \sum_{j=0}^{N} y_{k}(i, j)$

This equation provides a measure of the reduced total energy detected from the camera sensor for a certain optical setup over a given exposure time. Similar to the calculation of the maximum pixel value $\mu_{y, \max }$ described above, this integral of the pixel intensity values $\mu_{y, \text { total }}$ also provides the sensor characteristics independent of the brightness distribution in the measurement image. Considering a known intensity distribution function, $\mu_{y, \max }$ can be converted into $\mu_{y, \text { total }}$ and vice versa. In this case, both characteristics are equally applicable for the subsequent modelling. However, the calculation of $\mu_{y, \text { total }}$ might be a little more robust for the characterisation of an image brightness distribution with a large variation of brightness values.

Due to the very large and strongly varying influence of the laser beam propagation within the fibre material, the sub-pixel evaluation of the laser spot is not considered in this paper. We assume here that the maximum of the laser spot is always directly on a single pixel. Obviously this does not correspond to reality, but the difference is negligible due to the strong optical influence of the fibre material. The following section explains the procedure for using the modified EMVA 1288 in combination with an appropriate Point Spread Function (PSF) for the reflected laser spot.

\subsection{Image plane photon distribution}

Within this section the Fisher Information is calculated based on the EMVA 1288 sensor model and an appropriate PSF. Therefore, it is required to model the laser spot which is reflected back from the fibre material. Afterwards, the probability density function of this reflected laser spot image on the sensor is determined. On this basis, the FIM is calculated.

To begin with, Eq. 20 represents the PSF of the reflected laser spot. As previously justified, this reflected laser spot is centred within the image.

$$
\begin{aligned}
q(x, y)= & \frac{1}{2 \pi \sigma_{g, x} \sigma_{g, y} \sqrt{1-\varrho^{2}}} \\
& e^{-\frac{1}{2\left(1-\varrho^{2}\right)}\left[\frac{x^{2}}{\sigma_{x}^{2}}+\frac{y^{2}}{\sigma_{y}^{2}}-\frac{2 \varrho x y}{\sigma_{x} \sigma_{y}}\right]}
\end{aligned}
$$

This indicates, that the distribution's centre point is aligned with the centre point $(0,0)$ of the observation. For a more realistic modelling we consider that the angles $\alpha$ and $\beta$ can rotate independently of each other. Thus, the laser spot can be shaped oval on the surface. For this reason a two-dimensional Gaussian function is applied here. The standard deviations $\sigma_{g, o}$ given in Eq. 21 specify the shape of the observed, reflected laser spot. This parameter consists of the actual standard deviation of the initially emitted laser spot $\sigma_{l a, o}$ as well as the additional beam propagation offset parameter $p_{b, m, o}$ from Eq. 8. This describes the beam propagation within the composite material and the re-emission at another near by position on the materials surface.

$$
\begin{aligned}
\sigma_{g, o} & =p_{b, m, o}+\sigma_{l a, o}, \text { with } \\
\sigma_{l a, o} & =\frac{F W H M_{o}}{2 \sqrt{2 \ln (2)}} \text { and } p_{b, m, o} \in \mathbb{R}^{+}
\end{aligned}
$$

On this basis, Eq. 22 specifies the photon distribution over the sensor image plane under consideration of a magnification factor M given in Eq. 1 from the optical setup. For this, $\theta$ defines the used parameter vector.

$$
\begin{gathered}
f_{\theta}=\frac{1}{M^{2}} q\left(\frac{x}{M}-x_{0}, \frac{y}{M}-y_{0}\right) \\
\stackrel{x_{0}=y_{0}=0}{\longrightarrow}=\frac{1}{M^{3}} q(x, y)
\end{gathered}
$$

The lens distortion at the edges of the image is less than $0.2 \%$ for the viewed wavelength range [52]. Additionally, the reflected laser spot is captured very close to the optical axis. Thus, asymmetries from the optical setup can be neglected. For this reason, the magnification in $\mathrm{x}$ and $\mathrm{y}$ direction should be very similar. Thus, the magnification $\mathrm{M}$ is the average of the magnifications in the $\mathrm{x}$ direction $M_{x}$ and in the y direction $M_{y}$. This leads to $M=\frac{1}{2}\left(M_{x}+\right.$ 
$M_{y}$ ). As explained above, we assume that the centre of the reflected laser spot is in line with the centre of the viewing area. This leads to $x_{0}=y_{0}=0$.

\subsection{Sensor data model}

Afterwards, the representation of the reflected laser spot on the image plane is utilised to determine its effect on each individual pixel. For this purpose, the random variable $H_{\theta, k}$ specifies the number of recognised electrons in each individual $\mathrm{k}$-th pixel of the viewing region:

$H_{\theta, k}=S_{\theta, k}\left(\mu_{\theta, k}\right)+B_{\theta, k}\left(\beta_{\theta, k}\right)+W_{\theta, k}\left(\mu_{q}, \sigma_{q}\right)$

For this , $S_{\theta, k}$ is an independent Poisson random variable which represents the photons converted into electrons. $B_{\theta, k}$ is an independent Poisson random variable which specifies the electrons caused from background noise in each pixel. In this case, the background noise describes the number of detected electrons without any incident light. Thus, in this paper, background noise consists only of sensor internal noise. Accordingly, this is equivalent to dark noise and does not refer to disturbing ambient lighting or similar. $W_{\theta, k}$ represents a Gaussian random variable which specifies the amount of electrons caused by readout noise in a pixel. Due to mathematical calculation rules for the Poisson distribution, for $S_{\theta, k}$ and $B_{\theta, k}$ can be stated that $\operatorname{Var}\left(S_{\theta, k}\right)=\mu_{\theta, k}$ and $\operatorname{Var}\left(B_{\theta, k}\right)=\beta_{\theta, k}$. In contrast, $W_{\theta, k}$ is Gaussian distributed with mean $\mu_{q}$ and $\operatorname{Var}\left(W_{\theta, k}\right)=\sigma_{q}^{2}$. $W_{\theta, k}$ is used in accordance with the readout noise from the EMVA 1288 model in Section 4.1. This EMVA 1288 model specifies the both statistical parameters. Concluding the mean number of electrons of the detected sensor signal for each k-th pixel $v_{\theta, k}$ is given by:

$v_{\theta, k}=\mu_{y}(k)=K \eta_{q e} \mu_{\theta, k}+\beta_{\theta, k}$

This consists of the actual information $\mu_{\theta, k}$ and some sensor internal background noise $\beta_{\theta, k}$. Accordingly, the readout noise is not part of $v_{\theta, k}$. The variable $\mu_{y}(k)$ was previously given from the EMVA 1288 Eq. 16 from Section 4.1.1. According to the properties of the Poisson distribution $\mu_{\theta, k}+\beta_{\theta, k}$ lead to $\operatorname{Var}\left(\mu_{\theta, k}+\beta_{\theta, k}\right)=\left(v_{\theta, k}+\right.$ $\beta_{\theta, k}$ ).

In this case $\beta_{\theta, k}$ in Eq. 25 is the mean number of counted electrons of the dark current of the image sensor. For this representation Eq. 16 from Section 4.1.1 is considered.

$\beta_{\theta, k}=K \mu_{d}=\mu_{y . d a r k}$

In accordance with Eq. 26, the signal intensity is distributed over the sensors image plane with mean $\mu_{\theta, k}$. More precisely, the average number of photons detected within the sensor region $C_{k}$ over the time interval $\left[t_{0}, t_{\text {exp }}\right]$ is shown.

$\mu_{\theta, k}=\int_{t_{0}}^{t_{e x p}} \Lambda_{\theta}(t) \int_{C_{k}} f_{\theta}(x, y) d x d y$

Under consideration of Eqs. 12 and 14 as well as reflectivity of the material from Section 3.1.1, $\mu_{\theta, k}$ is calculated as:

$$
\begin{aligned}
\mu_{\theta, k} & =\mu_{p, \max } f_{\theta}(x, y) \\
& =\frac{C}{2 M^{3} \pi \sigma_{g, x} \sigma_{g, y} \sqrt{1-\varrho^{2}}} \mu_{p, \max } \\
& =\frac{t_{\exp } \lambda_{l a}}{h c} \frac{A_{p x} C}{2 M^{3} \pi \sigma_{g, x} \sigma_{g, y} \sqrt{1-\varrho^{2}}} E_{p x, \max } \\
& \text { with } C=e^{-\frac{1}{2\left(1-\varrho^{2}\right)}\left[\frac{x^{2}}{\sigma_{g, x}^{2}}+\frac{y^{2}}{\sigma_{g, y}^{2}}-\frac{2 \varrho x y}{\sigma_{g, x} \sigma_{g, y}}\right]}
\end{aligned}
$$

As mentioned above, considering a Gaussian beam profile with centre $(0,0)$ gives $E_{p x, \max }=E_{l a}(0,0) \varrho_{m} \varrho_{s} \varrho_{a} \varrho_{f}$. The corresponding reduction factor $\varrho_{i}$ are introduced above in Section 3.1 and Section 4.1.1. For the following calculations we assume, that $\sigma_{g}=\sigma_{g, x}=\sigma_{g, y}$. For this, we first assume, $\sigma_{l a}=\sigma_{l a, x}=\sigma_{l a, y}$. This is valid due to the marginal tilting of the laser and camera as well as small laser beam variance compared to the beam propagation of the fibre material. Furthermore, we assume a direction invariant beam propagation within the material $p_{b, m}=p_{b, m, x}=$ $p_{b, m, y}$.

Due to the anisotropic composite material and the fibre angle dependent reflection behaviour, as already described from Stokes-Griffin and Compston [43], in reality the beam propagation will probably vary with the fibre orientation. Additionally, laser speckle can lead to a jagged contour of the laser spot in reality. However, since we only consider a very small laser spot projected which is detected almost perpendicularly, such effects were neglected here. If the laser spot is projected and detected at a large angle, this assumption must be reconsidered.

However, the focus of this paper is primarily on proving the basic validity of the proposed model. In order to keep the calculations as comprehensible as possible, this assumption is applied despite the potential deviation from a realistic beam propagation.

Hence, it is possible that the laser spot has a slightly oval shape in reality, whereas in the calculation the spot is ideally circular. However, the peak value should be only slightly affected by this assumption, as the input energy is not influenced by the ratio of the direction-dependent beam propagation.

In the investigations in this paper, we are checking the potential error for this assumption and examine if an 
adjustment is necessary. Thus, this assumption needs to be taken into account in the evaluation of the model. With this information, Eq. 7 from Section 3.3 can be applied. Accordingly, $\mu_{\theta, k}$ can be written as:

$$
\begin{aligned}
\mu_{\theta, k}= & \frac{1}{2 M^{3} \pi \sigma_{g}^{2}} \mu_{p, \text { max }} e^{-\frac{x^{2}+y^{2}}{2 \sigma_{g}^{2}}} \\
= & \frac{t_{\exp } \lambda_{l a} A_{p x}}{2 h c M^{3} \pi\left(p_{b, m}+\sigma_{l a}\right)^{2}} E_{p x, \text { max }} \\
& e^{-\frac{x^{2}+y^{2}}{2\left(p_{b, m}+\sigma_{l a}\right)^{2}}}
\end{aligned}
$$

Applying the previous assumptions for $\sigma_{g}$ and $p_{b, m}$ leads to Eq. 29 for the mean detected sensor signal $v_{\theta, k}$ for the k-th pixel.

$$
v_{\theta, k}=K\left(\eta_{q e} \frac{t_{\exp } \lambda_{l a} A_{p x}}{2 h c M^{3} \pi \sigma_{g}^{2}} E_{p x, \max } e^{-\frac{x^{2}+y^{2}}{2 \sigma_{g}^{2}}}+\mu_{d}\right)
$$

Taking the previous calculations into account, Eq. 30 presents the accumulated Probability Density Function (PDF) $\rho_{\theta, k}(z)$ for the accumulated signal and noise random variable $H_{\theta, k}$ from Eq. 23 with respect to an individual pixel $\mathrm{k}$.

$$
\begin{gathered}
\rho_{\theta, k}(z)=\frac{e^{-v_{\theta, k}}}{\sqrt{2 \pi \sigma_{q}^{2}}} \sum_{l=0}^{\infty} \frac{v_{\theta, k}^{l}}{l !} e^{-\frac{\left(z-l-\mu_{q}\right)^{2}}{2 \sigma_{q}^{2}}} \\
, z \in \mathbb{R}, \theta \in \Theta
\end{gathered}
$$

As previously introduced, the readout noise $\mu_{q}$ is Gaussian distributed over the considered pixel region. In contrast, the signal as well as background noise are Poisson distributed across the evaluated pixel. $\mathrm{z}$ represents the photon counter. $v_{\theta, k}$ gives the mean for the Poisson distributed photon hits within the signal $\mu_{\theta, k}$ including background noise $\beta_{\theta, k} . \mu_{q}$ represents the mean of the corresponding Gaussian distributed readout noise per pixel with variance $\sigma_{q}^{2}$. The signal and the readout noise can be assumed to be independent random variables. This implies that the convolution of both PDFs is expressed as a multiplication of the individual PDFs. This formulation is inspired from Chao et al. [7].

Compared to the laser peak signal $\mu_{\theta, k}$ the readout noise $\mu_{q}$ is very small. For this particular case, if $\mu_{q}<<\mu_{\theta, k}$ we can assume that $\mu_{q}=0$. Applying this assumption leads to the following PDF for each $\mathrm{k}$-th pixel:

$\rho_{\theta, k}(z)=\frac{v_{\theta, k}^{z} e^{-v_{\theta, k}}}{z !}$

This equation is used for the FIM calculation in the following section.

\subsection{Calculation of Fisher Information and Cramér-Rao bound}

Subsequently the general form of the FIM for some observed data is given by:

$\mathbf{I}(\theta)=-\mathbf{E}\left[\frac{\partial^{2} \ln \rho_{\theta}(\omega)}{\partial \theta_{i} \partial \theta_{j}}\right]$

In this equation $\omega$ represents the considered data with the corresponding PDF $\rho_{\theta}(\omega)$ and a given parameter vector $\theta$ [62]. Thus, this Eq. 32 presents the derivative of the $\log$ likelihood function in relation to $\theta$ for each individual pixel [7]. Derived from this, the FIM over multiple pixels can be calculated from the given PDF from Eq. 31. On this basis the accumulated FIM over all $\mathrm{Z}$ pixels of the considered sensor area is represented through:

$\mathbf{I}(\theta)=\sum_{k=1}^{Z} \mathbf{E}\left[\left(\frac{\partial \ln \rho_{\theta, k}(z)}{\partial \theta}\right)^{\top}\left(\frac{\partial \ln \rho_{\theta, k}(z)}{\partial \theta}\right)\right]$

For the typical form of this estimation problem, $\theta$ parametrises just the true value $v_{\theta, k}$ in the $\mathrm{k}$-th pixel of the Poisson component of the data. Thus, the corresponding FIM can be written as:

$\mathbf{I}(\theta)=\sum_{k=1}^{Z}\left(\frac{\partial v_{\theta, k}}{\partial \theta}\right)^{\top}\left(\frac{\partial v_{\theta, k}}{\partial \theta}\right) \mathbf{E}\left[\left(\frac{\partial \ln \left(\rho_{\theta, k}(z)\right)}{\partial v_{\theta, k}}\right)^{2}\right]$

This formulation of the FIM is inspired by Chao et al. [7]. In this case the FIM for the entire viewing area is calculated from the sum of the individual FIMs of each k-th pixel. Subsequently, the score function for an unbiased estimator is given by:

$S\left(v_{\theta, k}\right)=\left(\frac{\partial \ln \left(\rho_{\theta, k}(z)\right)}{\partial v_{\theta, k}}\right)^{2}$

Considering the properties of a Poisson distribution the Fisher Information of $v_{\theta, k}$ is represented as:

$I\left(v_{\theta, k}\right)=\operatorname{Var}\left(S\left(v_{\theta, k}\right)\right)=\frac{1}{v_{\theta, k}}$

Evaluating Eq. 34 with the Fisher Information for $v_{\theta, k}$ from Eq. 36 results in the following FIM:

$\mathbf{I}(\theta)=\sum_{k=1}^{Z}\left(\frac{\partial v_{\theta, k}}{\partial \theta}\right)^{\top}\left(\frac{\partial v_{\theta, k}}{\partial \theta}\right) \cdot \frac{1}{v_{\theta, k}}$

Subsequently, the CRLB can be calculated on basis of the FIM. This CRLB describes the lower bound to any unbiased estimator for our problem. It can be derived by evaluating the inverse of the FIM, which is widely interpreted as a kind of covariance matrix. Thus, the CRLB can be used to obtain 
the performance on the estimator used in an experiment. If the experimental results are close to the CRLB for long data sequences the estimator is called efficient [62]. In this case the estimator is optimal in terms of accuracy. The diagonal elements of the following inequality represent the CRLB values.

$\operatorname{var}(\hat{\theta}) \geq I(\theta)^{-1}$

In order to illustrate the CRLB results in this paper, they are exemplary calculated for $\mu_{p, \max }$ and $\sigma_{g}$. Then they are plotted with respect to the exposure time and the number of incident photons. Thus, the parameter vector is specified as $\theta=\left\{\mu_{p, \max }, \sigma_{g}\right\}$.

The following section explains the simulative calculation of a laser spot image according to the previously introduced model.

\section{Simulation of the incoming photons and their processing}

Within this section the sequence of the implemented simulation is presented. This serves to simulate the image of the reflected spot laser on the camera sensor and the corresponding output signal. This simulation is based on the sensor model and the corresponding PSF from Section 4 for the spot laser specified in Section 3.1.

Since this simulation is intended to pragmatically reproduce a recorded real laser spot quite well, assumptions which are necessary for the previous statistical evaluation were slightly modified for an improved simulation outcome.

The simulation was implemented in Python 3.8.4. Additionally, the libraries numpy 1.19.0, matplotlib 3.2.2 and scipy 1.5.0 were used. All calculations were performed sequentially on the Central Processing Unit (CPU). Furthermore the input and output of the simulation as well as deviations from the modelling in Section 4 are explained below.

(1) Input: The simulation receives all relevant characteristic values from the sensor system, lens, material and laser beam as input. In addition, the exposure time and sensor amplification are necessary. All the corresponding parameters are presented for the experimental setup in Section 3.1. The sensor properties consist of the quantum efficiency $\eta_{q e}$, the mean dark noise $\mu_{d}$, as well as mean value and standard deviation of the readout noise $\mu_{q}, \sigma_{q}$. Furthermore the area of a single pixel $A_{p x}$ is required. This is calculated from the pixel dimensions $s_{p x, x}$ and $s_{p x, y}$. As already stated in Section 3.1, the fill factor for the installed sensor is almost $100 \%$. Due to this reason the fill factor is assumed to be one in this simulation and is not considered any further.
The lens and optical characteristics consist of the adjusted aperture f-number $f_{a}$, the focal length $\mathrm{f}$ of the lens, the absorption coefficient of the built-in filter $\varrho_{f}$ and the working distance $d_{w}$ of the lens. The corresponding values and relationship between these influencing factors are introduced in Section 3.1. Due to Eq. 12 the relative reflectivity of the material $\varrho_{m}$ is required. Furthermore in accordance with Eq. 8, the beam propagation parameters $p_{1}, p_{2}$ are utilised. The laser spot is defined through the laser wavelength $\lambda_{l a}$, laser power $P_{L}$ and the FWHM of the Gaussian laser profile in $\mathrm{x}$ and $\mathrm{y}$ direction. These values are utilised in Eqs. 11 and 14 and introduced in Section 3.1.

(2) Approximated top hat profile: Following the characteristics of a reflected laser beam profile introduced in Section 2.2.1 from Stokes-Griffin and Compston [43], a top hat like laser profile is enforced for the applied simulation.

At this point, the assumption of a Gaussian intensity profile from the calculations in Section 4 is extended.

Accordingly, we assumed that values above a certain intensity level result in a nearly homogeneous part of the sensor image. The case diffraction in Eq. 39 expresses this. The corresponding top hat maximum value in Eq. 40 is specified according the function in Eq. 9 of the laser beam profile. The parameter $r_{T H}$ defines the width of the top hat plateau in the beam profile. Considering the originally Gaussian beam profile, this value is chosen to be quite small in this paper $r_{\mathrm{TH}}=\sqrt{\frac{1}{8}}$.

$\mu_{y}(i, j)= \begin{cases}\mu_{y, \max , \mathrm{TH}} & \mu_{y}(i, j)>\mu_{y, \max , \mathrm{TH}} \\ \mu_{y}(i, j) & \text { other }\end{cases}$

$\mu_{y, \max , \mathrm{TH}}=\frac{1}{\exp \left(2 \frac{r_{\mathrm{TH}}^{2}}{w^{2}}\right)}=\frac{1}{\exp \left(\frac{1}{4}\right)}$

(3) Image visualisation and output: The simulated reflected laser spot images are first exported as 16-bit greyscale Tagged Image File Format (TIFF) images. Those serve a comparable evaluation of the real measurements and the simulated data. Then these simulated images are applied to the same evaluation chain than the real measurement images. Accordingly, the simulated reflected laser spot images are evaluated with the identical methods as the real measurement images.

The procedure of a simulation run is illustrated in Fig. 6. The corresponding implemented equations are given at the end of each box.

In the following section, the results of the simulation are compared to the real measurement images. Therefore, the performance of the simulation and the deviations from the real measured reflected laser spot images are examined. Furthermore, the captured laser spot images are reviewed. 
(1) Input parameters

(2) Estimate beam propagation factor $p_{b, m}$ [Eq. 8]

(3) Generate number of incident photons for laser irradiance $E_{l a, \text { Gaus }}$ [Eq. 9]

(4) Estimate laser speckle factor $\varrho_{s}$ [Eq. 13]

(5) Calculate amount of photons incident on sensor $\mu_{p}$ [Eq. 12]

(6) Approximate photon distribution on sensor $f_{\theta, \tau}$ [Eq. 22]

(7) Estimate amount of sensor electrons $\mu_{e}$ from incident photons $\mu_{p}$ [Eq. 15]

(8) Apply dark noise $\mu_{d}, \sigma_{d}$ and gain $\mathrm{K}$ [Eq. 16]

(9) Apply quantisation noise $\mu_{q}, \sigma_{q}$ [Eq. 16]

(10) A/D conversion and result image $\mu_{y}(i, j)$ generation [Eq. 16]

(11) Generate Top Hat image from case differentiation [Eq. 39]

(12) Image visualisation and output

Fig. 6 The flow chart illustrates the sequence of the simulation. The steps are sequentially numbered. The corresponding and implemented equations for each simulation step are given

\section{Results}

In this section, initially the reflection and transmission properties of the fibre composite material are presented. Then the results of the laser spot measurements are compared to the simulated laser spot data.

\subsection{Beam propagation parameters estimation}

This section presents the approximated results for the beam propagation parameters $p_{1}$ and $p_{2}$ from Eq. 8. The corresponding methodology was discussed in Section 3.3. In conclusion, an overview of all applied simulation parameters is shown. This serves as the basis for the subsequent validation experiments.

The corresponding results from the estimation of $p_{1}$ and $p_{2}$ are presented in Fig. 7 .

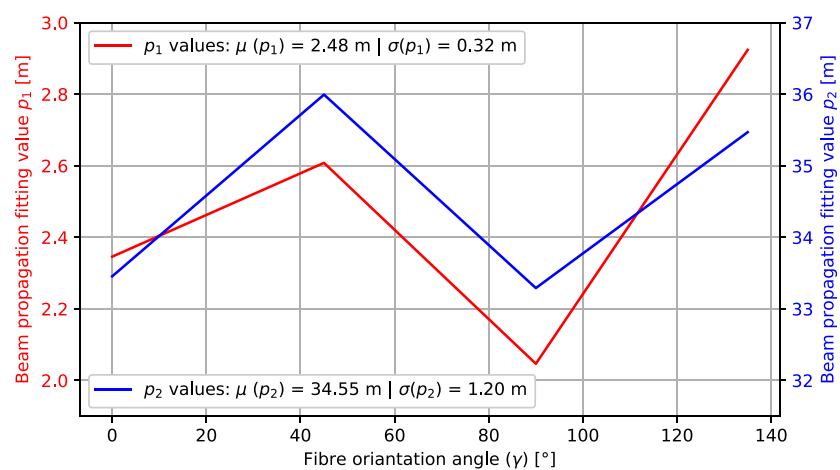

Fig. 7 Plot of the approximated beam propagation parameters $p_{1}$ and $p_{2}$ for different fibre orientations $\gamma$. The mean values and the standard deviations are given in the legend for each parameter. $p_{1}$ and $p_{2}$ are given in metre

The mean values for all measurements $\mu\left(p_{i}, \gamma\right)$ per rotation angle $\gamma$ are plotted for both parameters $p_{1}$ and $p_{2}$ on the two ordinates. The total mean of all measurements $\mu\left(p_{i}\right)$ and the corresponding standard deviation $\sigma\left(p_{i}\right)$ are presented in the legend. The two overall mean values $\mu\left(p_{1}\right)=2.48 \mathrm{~m}$ and $\mu\left(p_{2}\right)=34.55 \mathrm{~m}$ are applied to all simulations in this paper. The standard deviations for both parameters $\sigma\left(p_{1}\right)=0.32 \mathrm{~m}$ and $\sigma\left(p_{2}\right)=1.20 \mathrm{~m}$ are quite small. This indicates a robust fitting process. Furthermore, this strengthens the validity of applying a direction independent beam propagation parameter $p_{b, m}$ as assumed in Section 4.3.

All corresponding parameters applied for the simulation are summarised in Table 3. The simulation parametrised in this way is used for the evaluation in this study. The italic parameters are estimated from experiments. All the other variables are given from the discussions in Section 3.1.

\subsection{Laser spot image evaluation}

Within this section we compare the output of the implemented simulation from Section 5 with the analysis results for the real reflected laser spot. As described above, both the real and the simulated camera image of the reflected and imaged laser spot are examined with the same methods and identical configurations.

In order to give a visual impression of the match of the simulation with a real reflected laser spot image, a simulated image and a captured reflection image are compared in Fig. 8. Furthermore, Fig. 8 panel c illustrates the overlapping of both images. However, it is noteworthy that this comparison serves only as an exemplary illustration. The integration time $t_{\exp }=0.9 \mathrm{~ms}$ is applied for this scenario. These images display the laser spot reflected from the fibre material and imaged in the camera. The significant deviation from an ideal Gaussian intensity profile can be attributed to the optical properties of the observed material. 
Table 3 The table summarises all input parameters for the applied simulation in this paper

\begin{tabular}{|c|c|c|c|c|}
\hline Parameter & Symbol & Value(s) & Unit & Ref. \\
\hline Focal length & $\mathrm{f}$ & 0.0502 & $\mathrm{~m}$ & {$[52]$} \\
\hline Pixel size $x$ & $s_{p x, x}$ & $5.5 \cdot 10^{-6}$ & $\mathrm{~m}$ & [46] \\
\hline Pixel size y & $s_{p x, y}$ & $5.5 \cdot 10^{-6}$ & $\mathrm{~m}$ & [46] \\
\hline $\mathrm{A} / \mathrm{D}$ conversion resolution & - & 10 & bit & [48] \\
\hline Dynamic range & - & 60 & $\mathrm{db}$ & [48] \\
\hline Sensor A/D conversion gain & $\mathrm{K}$ & 0.11 & $\frac{D N}{e}$ & [48] \\
\hline Sensor quantum efficiency & $\eta_{q e}$ & 38 & $\%$ & {$[46,47]$} \\
\hline Dark noise & $\mu_{d}$ & 70 & $\frac{e}{s}$ & [48] \\
\hline Readout noise (mean) & $\mu_{q}$ & 26 & $\frac{e}{s}$ & [6] \\
\hline Readout noise (STD) & $\sigma_{q}$ & 408.33 & $\frac{e^{2}}{s^{2}}$ & [6] \\
\hline Laser power & $P_{L}$ & 0.001 & W & [45] \\
\hline Laser profile FWHM $-\mathrm{x}$ & $\mathrm{FWHM}_{l a, x}$ & $72.8 \cdot 10^{-6}$ & $\mathrm{~m}$ & [45] \\
\hline Laser profile FWHM - y & $\mathrm{FWHM}_{l a, y}$ & $80.4 \cdot 10^{-6}$ & $\mathrm{~m}$ & [45] \\
\hline Laser wavelength & $\lambda_{l a}$ & $6.5 \cdot 10^{-7}$ & $\mathrm{~m}$ & [45] \\
\hline Working distance & $d_{w}$ & 0.25 & $\mathrm{~m}$ & \\
\hline Aperture f-number & $f_{a}$ & 11 & - & \\
\hline Image width & - & 240 & $\mathrm{px}$ & \\
\hline Image height & - & 240 & $\mathrm{px}$ & \\
\hline Exposure time & $t_{\text {exp }}$ & $\{0.0001,0.0005,0.0009\}$ & s & \\
\hline Top hat factor & $r_{T H}$ & $\sqrt{\frac{1}{8}}$ & - & \\
\hline Material reflection & $\varrho_{m}$ & 2 & $\%$ & \\
\hline Beam propagation factor & $p_{1}$ & 2.48 & $\mathrm{~m}$ & \\
\hline Beam propagation offset & $p_{2}$ & 34.55 & $\mathrm{~m}$ & \\
\hline
\end{tabular}

The corresponding values and their units are given. Additionally, the literature references are provided, unless the values are defined or experimentally determined in this paper. The italic entries indicate that these are experimentally estimated material parameters

Nevertheless a 2D Gaussian approximation of the reflected laser spot beam profile is reasonable, due to the transferability of the presented approach to other materials. We need to consider, that the material is illuminated with a Gaussian intensity profile. Beyond that the top hat plateau of the reflected laser spot can be significantly smaller for different fibre materials. In such cases, the match with a Gaussian reflection profile is much larger. Moreover, the displacement of the laser spot from the image centre is related to the thickness of the specimen holder and minor geometric deviations in the fibre material. However, these effects can be neglected, since the laser spot is characterised through a Gaussian fitting over all pixels of the region of interest. Furthermore, the optical influences of the fibre material are assumed to be very large. Thus, it is assumed that minor deviations from the optical viewing axis have only marginal impact on the results.

We clearly see that the simulated top hat plateau fits very closely with the real laser spot image. However, the true laser spot image drops significantly steeper at the edge than the simulated measurement image. This results from our simulation assumption of a Gaussian intensity distribution with artificially generated top hat behaviour. We have to keep in mind that the calculations and analyses in this paper are based only on abstract parameters of the intensity distribution and not on the actual image of the laser spot. Accordingly, the deviations of the simulation in this region do not cause major concerns. Furthermore, we see regularly horizontal lines on the top hat plateau of the real laser spot image in Fig. 8b. These small intensity variations presumably resulted from interference phenomena mentioned above. Obviously, these intensity fluctuations have an influence on the maximum value of the laser spot. Here, we assume that the top hat plateau consists of all pixels with an intensity value greater than half the image maximum. Furthermore, all image pixels with less than $2 \%$ intensity drop from the image maximum belong to the intensity peaks in a measurement image. In this scenario, the ratio of peak to top hat pixels is between 1 and 5\% for the measured images. For this reason, they are negligible compared to the overall intensity level of the top hat plateau. This confirms the assumption previously made 


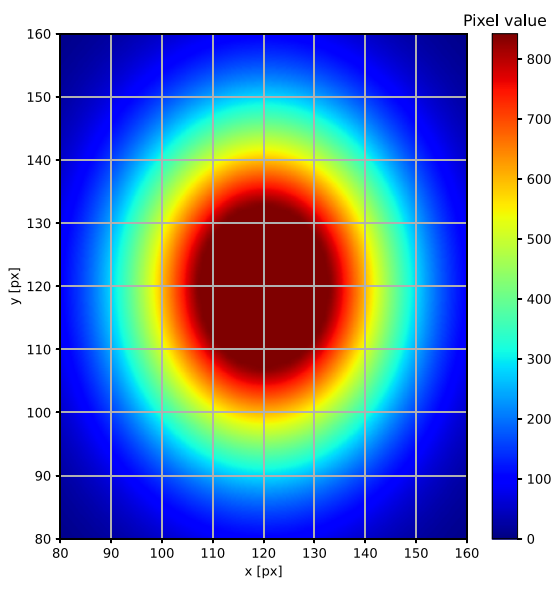

(a)

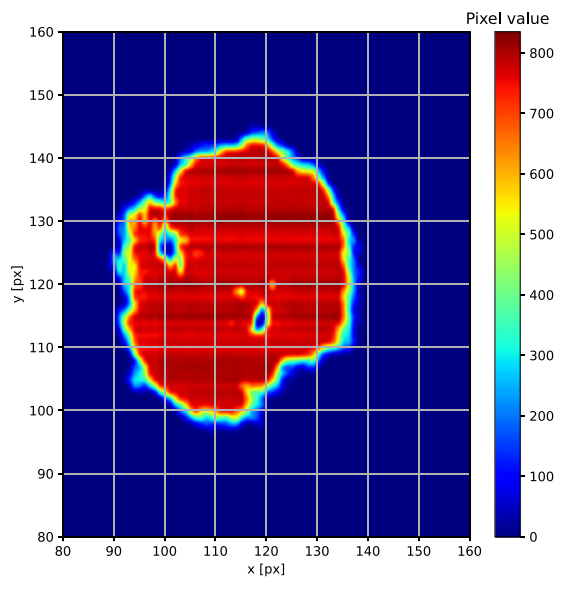

(b)

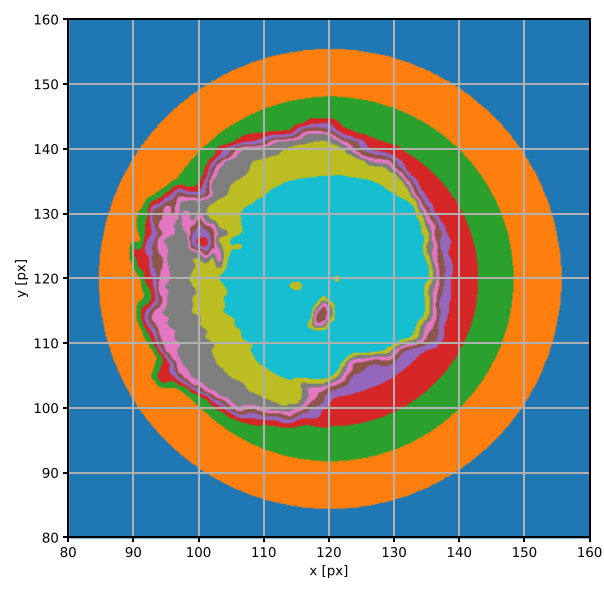

(c)

Fig. 8 Comparative illustration of the simulated and the recorded laser spot images for $t_{\text {exp }}=900 \mu s$. (a) Simulated laser spot image. A Gaussian intensity profile and an enlarged standard deviation with respect to the inter material beam propagation are applied. (b) Exemplary captured reflected laser spot image. Therefore, the

that interference effects do not need to be investigated in detail.

\subsubsection{Peak Signal-to-Noise Ratio analysis}

Initially, we consider the PSNR metric for the laser spot analysis. As stated above in Section 3.2, PSNR is the most promising for analysing white image noise [12, 58]. Subsequently, we examined the feasibility of characterising laser speckle and slight intensity variations on the laser spot as such a type of image noise via PSNR. The corresponding results are presented in Fig. 9.

Figure 9a shows the calculated PSNR mean values for the comparison of the simulated reference images and the corresponding real reflected laser spot images for different fibre orientations $\gamma$. The error bars represent their standard deviations. The mean values of the concatenated PSNR are in the interval $[53,58] \mathrm{dB}$. The corresponding standard deviations are in the interval $[0.7,3.4] \mathrm{dB}$. This clearly illustrates that the greatest PSNR values are obtained for the shortest exposure time and vice versa. The standard deviation, on the other hand, is quite similar for all exposure times.

For the interpretation of the PSNR larger values indicate lower noise and the other way around. Consequently, the PSNR values can be interpreted to represent less noise or intensity fluctuations on the top hat plateau of the laser spot for a shorter exposure time. This is certainly a plausible explanation, as disturbances and intensity fluctuations increase with longer exposure times. Furthermore, we notice that the graphs with potentially sufficient exposure times $t_{\text {exp }}=500 \mu s$ and $t_{\exp }=900 \mu s$ are relatively close

Picotronic DC650-1-3(8x25)-C500 laser illuminates the composite materials which reflects the beam to the AT C5-4090 camera sensor. (c) Superposition of the simulated laser spot image (a) and the actually recorded image data (b)

to each other. Both graphs are within the range of their own standard deviations. The red curve with a potentially insufficient exposure time of $t_{\text {exp }}=100 \mu \mathrm{s}$ is beyond the standard deviations of the other two plots except for $\gamma=$ $90^{\circ}$. Thus, these PSNR values can possibly be an additional indicator for the evaluation of a reasonable exposure time for this application. However, with the data evaluated in this paper, we cannot prove that the effects described above are significant and that they will appear reliably for other configurations. Further investigations were not carried out since the focus of the this study is on the overall assessment and modelling the given assembly.

In Section 3.2 we presented the expected value range for the calculated PSNR values for a proper correlation between the simulated and the real recorded laser spot measurement image. This range should be between 50 and $60 \mathrm{~dB}$ for the available data. Even taking the standard deviation into account, the PSNR values calculated here are well within this expected range of values. Thus, despite visual deviations, the simulated image correlates comparatively closely with the captured laser spot images.

\subsubsection{Investigation of laser intensity fluctuations}

Another scoring metric especially developed for the analysis of laser spots is the spatial speckle contrast value $C_{S}$ previously introduced in Eq. 2 from Section 2.2.1. We applied this metric to the captured image of the entire laser spot reflected from the composite. Applying this metric to the full image and not to small regions of the image enables the investigation of laser spot intensity fluctuations. Hence, the laser speckle as well as intensity fluctuations due to 


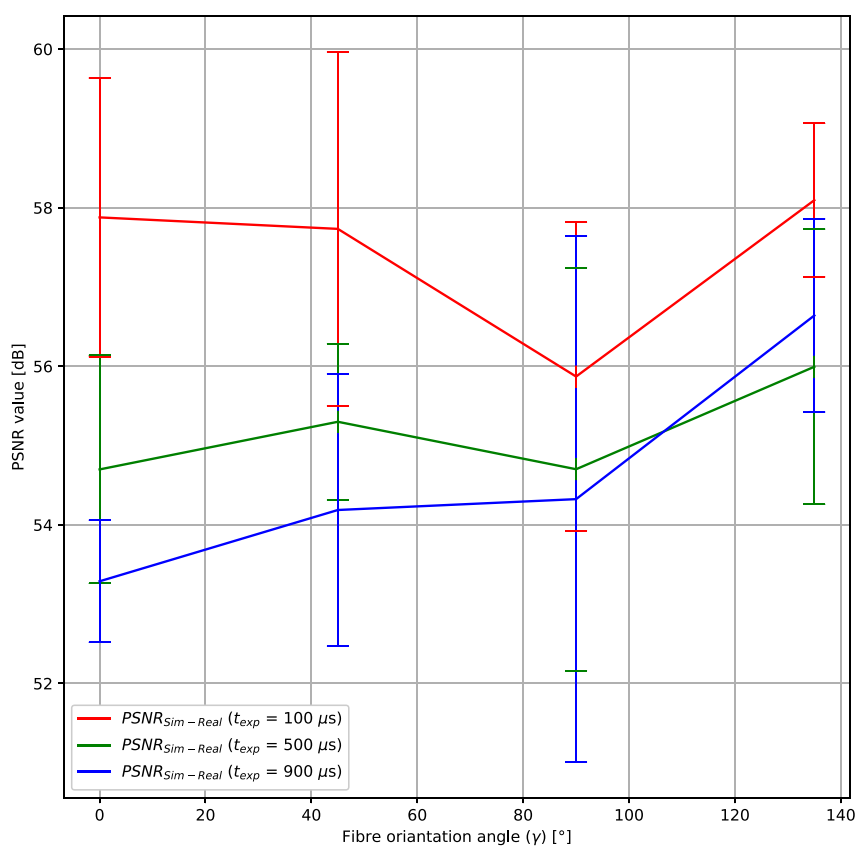

(a)

Fig. 9 The figure presents the results for the assessment metrics PSNR and spatial speckle contrast $C_{s}$ on the entire laser spot image. The findings for different exposure times $t_{\text {exp }}$ and various rotation angles $\gamma$ are compared for the simulation and the recorded measured data. The corresponding standard deviations given. (a) Peak Signal-to-Noise Ratio

near-surface beam propagation in the material are examined. The corresponding results for the measurements and simulations in this paper are shown in Fig. 9b. $C_{S}$ is given in px on the ordinate and the corresponding sample rotation $\gamma$ on the abscissa. As already explained above, the simulation does not consider sample rotation. Thus, the simulated laser intensity fluctuations are assumed constant for all $\gamma$. Obviously this constant value does not very accurately represent reality. However, this simulated value mainly serve to provides a reference to evaluate the $C_{S}$ values calculated for the real measurement images. Thus, this is adequate for the level of detail investigated here. To analyse the laser intensity fluctuation, this modified spatial speckle contrast is the standard deviation of the laser spot image in relation to its mean image intensity. The value range for the real reflected laser spot image is between $4.4 \mathrm{px}$ and 9.5 px with a standard deviation between 0.35 px and 1.8 px. However, we like to clarify that the speckle properties of the laser also depend on the bandwidth and coherence length of the laser. Nevertheless, in this study we intend to only superficial analyse the suitability of this metric for assessing the quality of the input signal. Furthermore, the entire reflected laser spot image is observed. Accordingly, also expanding regions with strongly varying laser intensities have an influence on the $C_{s}$ value. The comparatively large beam propagation close to the surface of the composite,

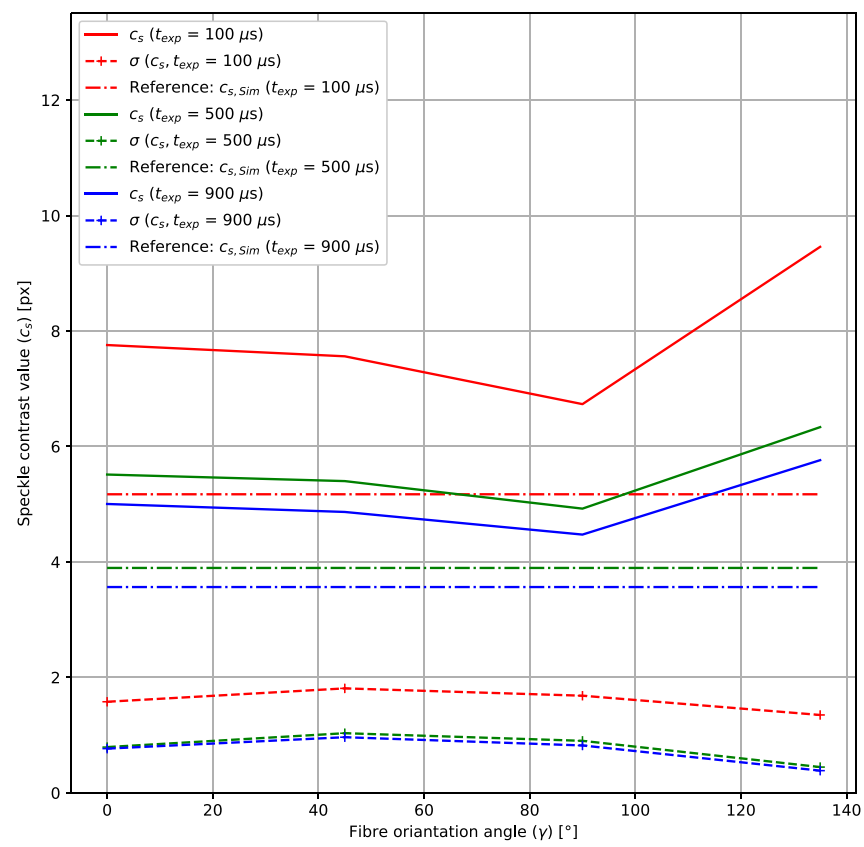

(b)

results for the comparison of the simulated and the real laser spot measurement image. (b) The figure illustrates the intensity fluctuation of the recorded laser spot image through the application of the spatial speckle contrast $C_{s}$ on the entire measurement image

which is illustrated in Fig. 7, has a great influence on the occurrence of these larger intensity fluctuations regions. The results for the simulated images are between $3.5 \mathrm{px}$ and $5.2 \mathrm{px}$. Considering the estimations of the aperture size $f_{a}$ from Section 3.1, the average diameter of a speckle grain $\bar{d}_{s}$ for $f_{a}=11$ is about 1.87 times the pixel size $s_{p x}$ which means $\bar{d}_{s}\left(f_{a}=11\right) \approx 1.87 \mathrm{px}$. This value provides a rough approximation of a theoretical error range for the simulated $C_{s}$ values. This error range closely corresponds to the maximum standard deviation of $1.8 \mathrm{px}$ of the measured speckle contrast values.

At first, we see that the $C_{S}$ value for the simulated laser spot is only slightly below the measurement results standard deviation. Furthermore, a value decrease for all measurements at $\alpha=90^{\circ}$ and a subsequent significant increase for the values between $\alpha=90^{\circ}$ and $\alpha=$ $180^{\circ}$ are evident. In addition, we observe a decrease in mean image intensity with lower exposure time, while the standard deviation remains relatively constant. Accordingly, this leads to higher laser intensity fluctuations at lower exposure times. This behaviour is also evident for the simulated values. We have to mention again that the $C_{s}$ value presented here contains the speckle of the laser as well as intensity fluctuations due to laser beam propagation and re-emission in the material. The beam profile and the intensity of the laser as well as the corresponding laser 
spatial speckle contrast remain constant. However, due to a variation in exposure time, varying amounts of photons are detected over the imaged reflected laser spot. This leads to a change in intensity fluctuation for the considered images of the entire reflected laser spot. Due to the $C_{s}$ values for the simulated images outlined above, the assumption of a Gaussian intensity profile on the image plane, as well as the top hat modification according to Eq. 39, is valid. Furthermore, the standard deviation of the speckle contrast $\sigma\left(C_{s}, t_{\text {exp }}\right)$ for $t_{\text {exp }}=100 \mu \mathrm{s}$ exposure time is about double the standard deviation for $t_{\text {exp }}=500 \mu s$ and $t_{\text {exp }}=900 \mu s$. This means that for $t_{\exp }=100 \mu \mathrm{s}$ very differently shaped reflected laser spot images are captured. Thus, this is an indication for an insufficiently short exposure time. In this case the laser spots can only be recorded sufficiently well under certain conditions. However, for the exposure times $t_{\text {exp }}=500 \mu s$ and $t_{\text {exp }}=900 \mu s$, the standard deviation $\sigma\left(C_{s}, t_{\text {exp }}\right)$ is significantly smaller and almost identical for all measurements. This metric is particularly well suited for the evaluation of the laser spot image quality for a given exposure time. However, this assessment is only valid if the entire laser spot is represented in the cropped image and the sensor avoids saturation.

\subsubsection{Top hat magnitude analysis}

Subsequently, the intensity maxima of the imaged reflected laser spots are analysed in Fig. 10. Such maxima are defined as input parameters for the EMVA 1288 modelling. Thus, they are suitable for the comparison of the simulated images with the real recorded measurement images. Figure 10a illustrates the actual pixel intensity maxima of the simulation and the average maxima of the measurements for each sample rotation angles $\gamma$. This serves to evaluate the top hat laser intensity level defined in Section 5. Figure 10b shows the maxima from the Gaussian curve fitting for both, the simulated and the real measurement images over the sample rotation angles $\gamma$. The standard deviations of the individual points are given as error bars. Again, the simulation is independent of the rotation angle $\gamma$ and thus forms a constant straight line.

We see in Fig. 10b that the top hat maximum values of the measured images are very close together for each exposure time and rotation angle. The maximum value difference is about 6 units, independent of the rotation angle or the exposure time. Thus, these maximum values appear to be a stable criterion for detecting a laser spot on a fibre composite material. Furthermore, the largest standard deviations at $\gamma=0^{\circ}$ and $\gamma=135^{\circ}$ are very small with $<10$ brightness units. The simulated maximum values deviate from the real average values by about 43 units in the worst case. For the two longer exposure times $t_{\text {exp }}=500 \mu \mathrm{s}$ and $t_{\text {exp }}=900 \mu \mathrm{s}$ this deviation is $<8$ units. This is in a similar range than the variation of the measured values for different rotation angles. Nevertheless, in comparison to the total maximum intensity of $>800$ units, this deviation is very small. Moreover, these small deviations between the simulation and the measured values indicate that the assumptions for the modelling of $\varrho_{s}$ in Eq. 13 are sufficient.

\subsubsection{Gaussian fitting amplitude evaluation}

Figure $10 \mathrm{~b}$ presents the calculated maximum values from the Gaussian curve fitting for both the simulated and the real measurement images. The individual values are again plotted over the rotation angles $\gamma$. Accordingly, error bars indicate the standard deviations. We see clearly that the calculated Gaussian maximum values vary significantly over different exposure times. For $t_{\text {exp }}=100 \mu \mathrm{s}$ the maxima are in the range of 839 to 924 brightness units. The brightness values are considerably larger for the two longer exposure times. They are between 992 and 1062 brightness units. In addition, the large standard deviation of at worst 227 units for $t_{\exp }=100 \mu \mathrm{s}$ is quite striking. In contrast, for the exposure times $t_{\text {exp }}=500 \mu s$ and $t_{\text {exp }}=900 \mu \mathrm{s}$ only a maximum standard deviation of the intensity values of 51 units is obtained. A possible reason for this major difference might be the sensitivity of the camera sensor. In case of $t_{\text {exp }}=100 \mu \mathrm{s}$ the incident irradiance of the sensor might drop below its sensitivity threshold. The large standard deviations for the measures for $t_{\text {exp }}=100 \mu \mathrm{s}$ indicate that the border areas of the laser spot are just at the sensitivity boundary of the camera sensor and thus the reflection is partly detected and partly rejected. In this respect we need to mention that no additional dark level threshold was set in the camera configurations. The corresponding "DarkLevelOffset" parameter [47] of the camera was set to zero. However, the findings about the meaning of the speckle contrast from Fig. $9 \mathrm{~b}$ are supported. This confirms that $t_{\text {exp }}=100 \mu \mathrm{s}$ is an insufficiently short exposure time for the application.

Apart from the presumably insufficient exposure time $t_{\text {exp }}=100 \mu \mathrm{s}$ the simulated maxima and the measured average maximum intensities differ less than 100 intensity units from each other. In this case the simulated maxima are larger than the measured values. Taking into account the top hat magnitudes from Fig. 10a, this indicates that the mathematical modelling of the input image and the curve fitting with a Gaussian function provide reasonable results. Nevertheless, a perceivable deviation from the actual laser spot image exists. This effect is qualitatively demonstrated in Fig. 8 for the simulated and an exemplary captured laser spot image. 


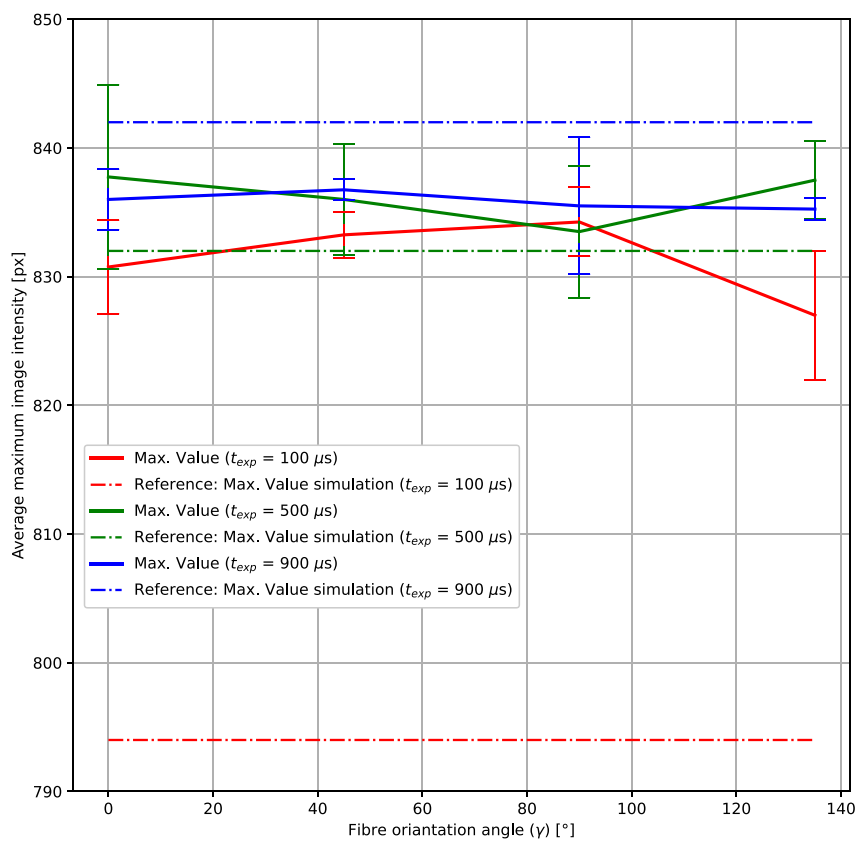

(a)

Fig. 10 The figure presents the actual top hat maxima and the approximated Gaussian amplitudes for the simulation and the real measurement images for different samples rotations $\gamma$ and the three examined integration times. The corresponding standard deviations are given

\subsubsection{Investigations on laser spot dimensions}

In accordance with the previously mentioned limitations of the applied mathematical model, we investigate the dimensions of the Gaussian laser spot approximation. Then we compare them to the simulated laser spot dimensions. For this reason Fig. 11 presents the directional independent average standard deviations from all 2D Gaussian curve fits. In this case, the standard deviation value does not represent a statistical parameter but the width of the approximated laser spot at a certain image intensity value. Thus, the width of the laser spot is analysed at a given position. These parameters are presented for all images of each corresponding fibre orientation angle $\gamma$. These values are compared to the directional and rotational independent laser spot width at standard deviation intensity level from the simulation $\overline{\sigma_{\text {Gaus,Sim }}}$. Again, the results for the three different exposure times are examined.

Initially we recognise that the $\overline{\sigma_{\text {Gaus }, \text { Sim }}}$ values for $t_{\text {exp }}=$ $500 \mu s$ and $t_{\text {exp }}=900 \mu s$ are more closely aligned than for $t_{\text {exp }}=100 \mu s$ and $t_{\text {exp }}=500 \mu s$ despite the identical exposure time difference of $\Delta t_{\text {exp }}=400 \mu s$. Moreover, a significant rise for the recorded images between $\gamma=45^{\circ}$ and $\gamma=90^{\circ}$ is noticeable. Afterwards the curves drop considerably towards $\gamma=135^{\circ}$. The standard deviations of the spot widths for different angles and exposure times are relatively similar. Their values range from $1 \mathrm{px}$ to $4 \mathrm{px}$. The

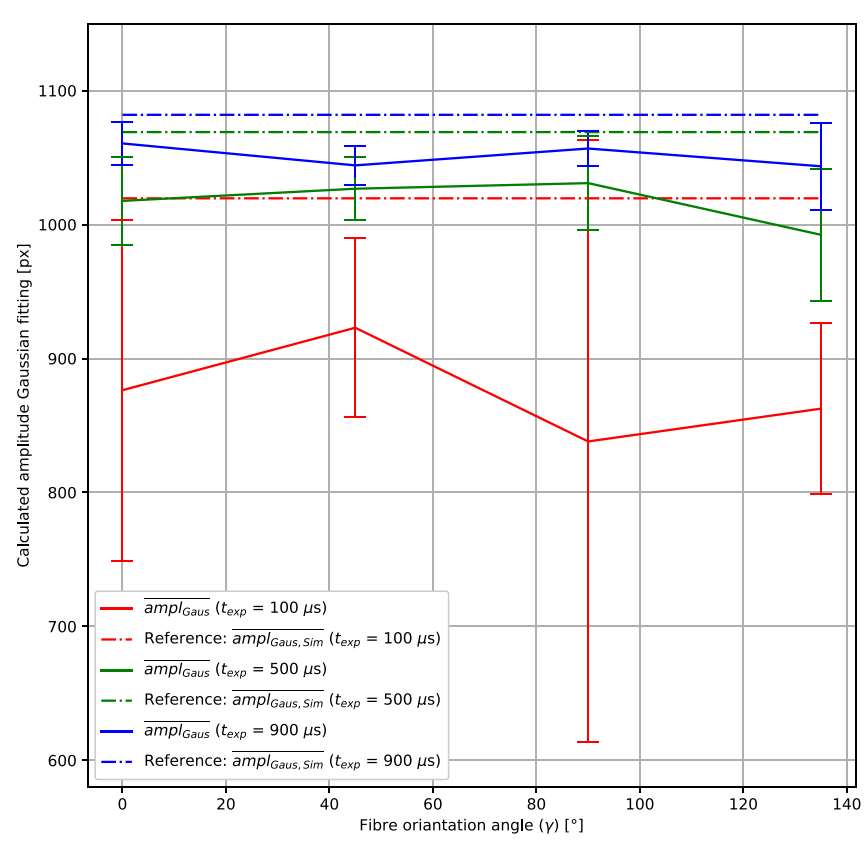

(b)

from the error bars. (a) Visualisation of actual measured and simulated maximum values of the reflected laser spot. (b) Amplitudes estimated from 2D Gaussian fitting for the simulation and the recorded laser spot images

simulated laser spot widths at standard deviation position correspond very well with the curve fittings of the measured laser spot profile. For the range between $\gamma=0^{\circ}$ and $\gamma=$ $90^{\circ}$ the maximum difference between the simulation and the fitted measurements is only about 2 px. For $\gamma=135^{\circ}$ and $t_{\exp }=100 \mu \mathrm{s}$ the difference is about $4.5 \mathrm{px}$. However, the standard deviation of the measured spot widths for this exceptional case is also around 4 px. We noticed that the simulated values are always within the standard deviation of the measured and fitted values or rather very close to it. This simulated average spot diameter at standard deviation position is based on the Gaussian laser beam profile and the beam propagation model from Eq. 8. Due to the perpendicular irradiation of the material and other major optical influences, no directional dependence of the material sample is considered for the simulated values. Further research is necessary to incorporate these directional influences into the simulation for non-perpendicular illumination. Consequently, the simulated values are valid for all fibre orientations $\gamma$ equally, but have an increased overall uncertainty. Furthermore is noteworthy that the given simulated reference values do not represent the average of the measured values. Due to the approach for the experimental determination of the beam propagation parameters $p_{1}$ and $p_{2}$ the simulated reference plots look very similar to the averaged measurement values. However, as outlined above, the simulated values match the measured values well for 


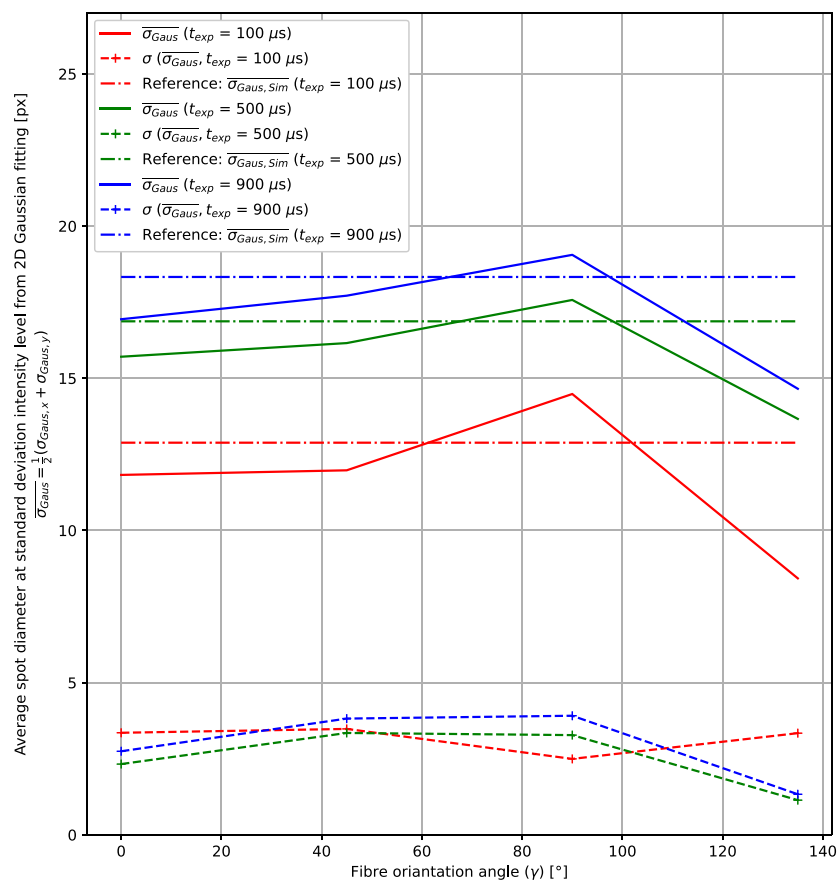

Fig. 11 Directional independent averaged diameter of the reflected laser spot at standard deviation intensity level, estimated from the 2D Gaussian laser spot fitting. The corresponding fitting values for the simulated and the captured images are compared and plotted for each sample rotation $\gamma$

different exposure times and fibre orientations. For this reason, we conclude that the simplified modelling of the beam propagation from Eq. 8 and the estimation of the model parameters $p_{1}$ and $p_{2}$ according to Fig. 7 allow a sufficiently accurate simulation.

\subsubsection{Analysis of the accumulated pixel values}

Finally, the integral of all pixel intensity values of the examined image regions, for the given fibre orientations $\gamma$ are analysed. This is shown in Fig. 12.

Accordingly, the corresponding standard deviations of the accumulated intensities are indicated with an error bar. For this, we have to remember that the image intensity values are directly correlated to the amount of incident photons. Consequently, we assume a linear response of the camera pixels. Thus, neither an intermediate fitting model nor other assumptions are made to calculate these values. With the exception of the integrals for $\gamma=$ $135^{\circ}$ and $t_{\text {exp }}=500 \mu \mathrm{s}$ at the position $\gamma=0^{\circ}$, all simulated accumulated values are within the standard deviation of the integrals from the measurement images. Nevertheless, we notice a slight increase of the simulated values compared to the average values of the measurement images. Furthermore, it is noteworthy that the standard deviations of the measured integral values up to 1.1 .

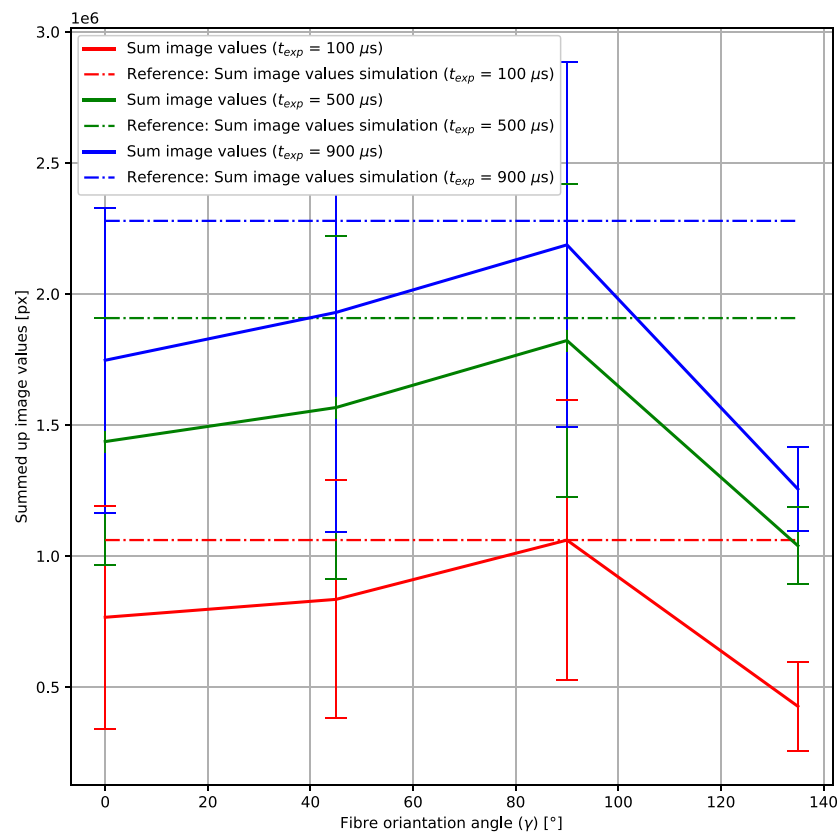

Fig. 12 Summed pixel values of the imaged reflected laser spot for different fibre orientations $\gamma$. The associated standard deviations are calculated from images with the same fibre orientation. The measured and the simulated values are presented

$10^{6} \mathrm{px}$ are relatively large. This effect is equally evident for all considered exposure times. This clearly indicates the varying appearance of individual laser spots in the measurement images. Thus, it is reasonable to carry out a model-based fitting of the measurement images in order to achieve evaluable results. This also justifies the Gaussian curve fitting for the investigations from above. Furthermore, this complex laser material interaction makes the modelling of the reflected laser spot in the measurement image more difficult. However, these sufficient simulation results confirm the assumption for the parameter $\varrho_{s}$ from Eq. 13 once more. Moreover, this standard deviation can also act as a kind of uncertainty indicator for the laser reflection. Thus, it also provides information about the precision of a depth measurement.

\subsubsection{Evaluation of the Fisher Information}

In the following Fig. 13 the calculated CRLB for $\theta=$ $\left\{\mu_{p, \max }, \sigma_{g}\right\}$ for the exposure times and photon counts are presented exemplary. The applied calculation parameters are listed above in Table 3.

As previously explained, the CRLB indicates the minimum variance for a certain parameter. Thus, we can determine the accuracy of estimating this particular parameter from a given model. The minimum variance for $\mu_{p, \max }$ is in the interval $\operatorname{Var}\left(\mu_{p, \max }\right)=\left[3 \cdot 10^{7}, 2.65 \cdot 10^{14}\right]$. For the minimum variance of $\sigma_{g}$ the value range $\operatorname{Var}\left(\sigma_{g}\right)=$ 


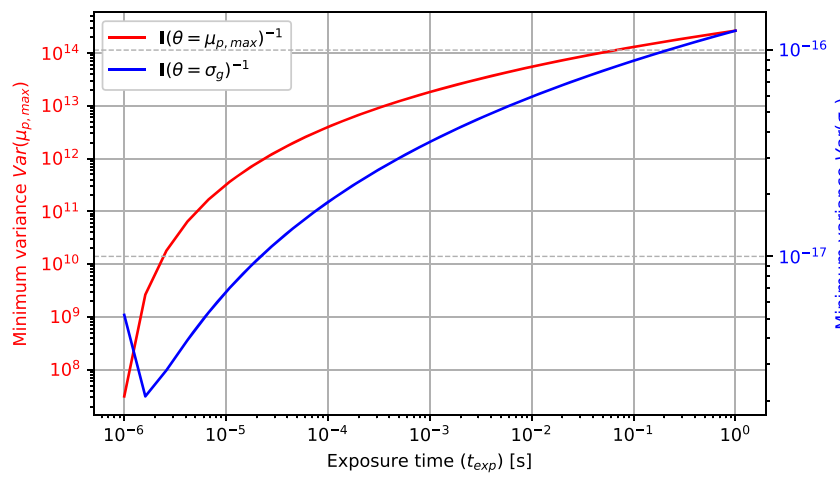

(a)

Fig. 13 Plot of the CRLB values of the number of photons as well as the expansion of the simulated reflected laser spot image over the exposure time on the left and number of incident photons on the right. (a) Plot of the CRLB values of the number of photons $\operatorname{Var}\left(\mu_{p, \max }\right)$

$\left[2 \cdot 10^{-18}, 1.25 \cdot 10^{-16}\right]$ applies for both plots. It is noteworthy that the variance of $\sigma_{g}$ is very small in each case. In contrast, for $\mu_{p, \max }$ the calculated variance is very large. This means that the number of photons $\mu_{p, \max }$ can be estimated with very large uncertainty. On the other hand, $\sigma_{g}$ can be estimated very well. Furthermore, we recognise that the minimum variance of $\sigma_{g}$ increases significantly in this model for very small exposure times. However, this is plausible but indicates a limitation of the beam propagation model from Eq. 8. For larger exposure times this minimum variance increases smoothly. For the variance of the number of incident photons $\operatorname{Var}\left(\mu_{p, \max }\right)$ a significant rise of the variance with increasing number of incident photons is evident in both plots. Concluding, it should be mentioned again, that the two parameters $\operatorname{Var}\left(\mu_{p}\right)$ and $\operatorname{Var}\left(\sigma_{g}\right)$ have only been examined exemplary to show the usability of the CRLB. For a more detailed investigation an extensive FIM and CRLB must be established and evaluated.

The results of this paper are discussed in the following section.

\section{Discussion}

This section discusses the major findings of this study and links them to the related research. In this paper different methods for modelling a sensor image and evaluating the corresponding data are investigated.

In the literature, Wang et al. [14] suggested a 2D Gaussian modelling for a laser beam reflected from CFRP material. This model corresponds rather accurately with the characteristics of the Gaussian approximated real reflected laser spot in this study. Accordingly, the assumption of a Gaussian beam profile for a reflected laser spot is valid for the simplified modelling in this study. However, in order to

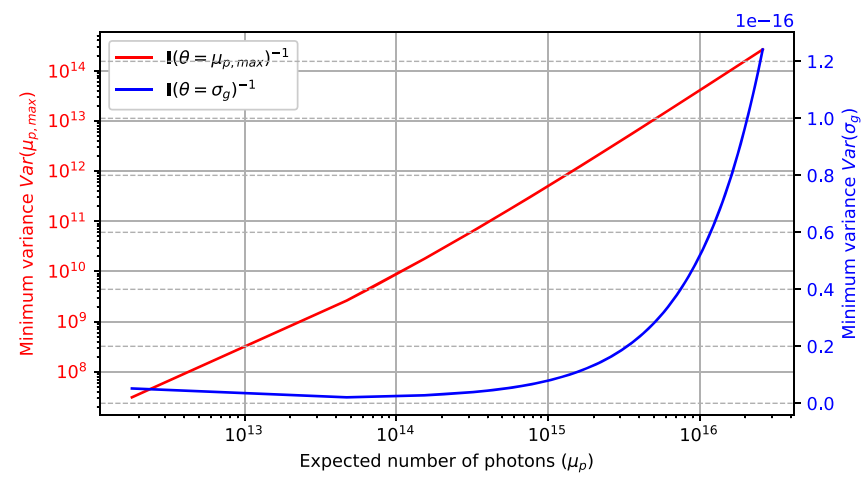

(b)

and the expansion of the simulated, reflected laser spot $\operatorname{Var}\left(\sigma_{g}\right)$ for various exposure times $t_{\text {exp }}$. (b) Plot of the CRLB values of the number of photons $\operatorname{Var}\left(\mu_{p, \max }\right)$ and the expansion of the simulated, reflected laser spot $\operatorname{Var}\left(\sigma_{g}\right)$ for different amounts of incident photons $\mu_{p}$

reproduce the top hat laser profile from Stokes-Griffin and Compston [43] an alternative beam modelling needs to be considered.

Furthermore, our results indicate that the camera sensor modelling according to the modified EMVA 1288 procedure yields reasonable results for a basic modelling of the considered test case. This answers the first research question. A more precise modelling of the reflected laser requires a more detailed description of the interaction of the laser beam with the fibre composite material. For the simulation of the reflected laser and the mathematical camera model, a top hat plateau is artificially formed as previously explained. Therefore, we assume that all values above the Gaussian beam profile position $r_{\mathrm{TH}}=\sqrt{\frac{1}{8}}$ equal the value of the Gaussian intensity profile at this position. Despite the simplicity of this hypothesis, our experimental results indicate their validity.

Referring to the second research question, we would like to summarise the informative value of the examined metrics for the application case under consideration. The PSNR primarily show the correspondence between the measured and the simulation images. The spatial speckle contrast of the entire laser spot image and the corresponding standard deviation are reliable measures for investigating the image intensity fluctuations. Thus, they are sensible to assess the sufficiency of the exposure times. In this case larger speckle contrasts and higher variances indicate an insufficient exposure time. The standard deviations of the approximated laser spot maximum values are a reasonable metric for assessing the laser spot quality. Since a robust laser spot shape leads to precise depth measures, this is implicitly a feasible metric for this scenario. In the performed experiments this standard deviation is quite large for the exposure time $t_{\exp }=100 \mu \mathrm{s}$. This means, that an exposure time of $t_{\text {exp }}=100 \mu \mathrm{s}$ is too short for 
this application case. Accordingly we carried out that the maximum value of the top hat plateau yield a stable measure for detecting the laser spot also for insufficient exposure times. Furthermore, it appears that the standard deviations of the accumulated pixel values can serve as an uncertainty parameter for the depth image estimation. Finally, the CRLB results seemed to be suitable to analyse the accuracy and limitations of the applied model. In this particular case these calculated values show a good predictability of the laser spot size but a poor estimability of the number of incident photons.

The approaches outlined in this study for modelling a sensor and evaluating signal quality can be applied in future research to evaluate the behavioural variation of detection and classification algorithms with changing signal quality. This further research is necessary to evaluate the impact of a varying signal quality on the defect detection and classification accuracy. Using the approaches from this study, the increase in efficiency of the inspection step during production can then be assessed in relation to the signal quality.

The following section summarises the findings from this paper and highlights the added value of our research.

\section{Conclusion}

In this section we summarise the key findings of this paper and highlight the contribution to industry and research. We have shown that the modelling of a Laser Line Scan Sensor with a modified European Machine Vision Association 1288 approach is suitable. Additionally this model is implemented in a simulation and is then compared to the experimental results in this study. For this purpose it is necessary to consider the perpendicular degree of reflection of the fibre material.

Furthermore, we demonstrated that the Gaussian laser spot fitting characteristics are also meaningful for a top hat profile. However, metrics such as speckle contrast are particularly well suited to simply assess the sufficiency of a configured exposure time. This procedure is also used to evaluate the presence of distinctive image characteristics. The presented Cramér-Rao lower bound provides a sensible way to evaluate the camera model. Here it is particularly noticeable that the the size of the laser spot can be estimated very well. On the other hand, the number of incident photons is rather difficult to estimate with this model.

The findings from this paper are very beneficial for developers of camera-based inspection systems for the Automated Fibre Placement process. Our findings support the material specific selection of a sensor and its optical design. Furthermore, the model implemented in the simulation serves to identify potential limitations of the inspection of a certain component prior to the actual production. We presented a model- and simulation-based approach for modelling a Laser Line Scan Sensor in this paper. With low effort this procedure and the presented evaluation methods can be transferred to other camera-based inspection and production processes. Therefore, the added value of this research is not limited to the fibre placement technology.

In future research the introduced spot laser model needs to be extended to the application of a line laser. In addition, the model should take movement influences during scanning at varying velocities into account. Furthermore, the analysis of the Fisher Information Matrix and CramérRao lower bound needs to be extended to determine an efficient estimator.

Moreover, the influence of the signal quality on the behaviour of the subsequent algorithms should be investigated in more detail.

Funding Open Access funding enabled and organized by Projekt DEAL. This research is part of the project DHiiP-AIR and was financially supported by the Federal Ministry for Economic Affairs and Energy. This project has received funding from the Federal Ministry for Economic Affairs and Energy under the funding code No. 20W1911F

Availability of data and material The corresponding image data is made available on request.

Code availability The corresponding Python code is made available on request.

\section{Declarations}

Conflict of interest The authors declare no competing interests.

Open Access This article is licensed under a Creative Commons Attribution 4.0 International License, which permits use, sharing, adaptation, distribution and reproduction in any medium or format, as long as you give appropriate credit to the original author(s) and the source, provide a link to the Creative Commons licence, and indicate if changes were made. The images or other third party material in this article are included in the article's Creative Commons licence, unless indicated otherwise in a credit line to the material. If material is not included in the article's Creative Commons licence and your intended use is not permitted by statutory regulation or exceeds the permitted use, you will need to obtain permission directly from the copyright holder. To view a copy of this licence, visit http://creativecommons. org/licenses/by/4.0/.

\section{References}

1. Marsh G (2010) Airbus a350 XWB update. Reinf Plast 54(6):2024. https://doi.org/10.1016/s0034-3617(10)70212-5

2. McIlhagger A, Archer E, McIlhagger R (2020) Manufacturing processes for composite materials and components for aerospace applications. In: Polymer composites in the aerospace industry, pp. 59-81. Elsevier. https://doi.org/10.1016/b978-0-08-102679-3. 00003-4 
3. Rudberg T, Nielson J, Henscheid M, Cemenska J (2014) Improving AFP cell performance. SAE Int J Aerosp 7(2):317-321. https://doi.org/10.4271/2014-01-2272

4. Eitzinger C (2019) Inline inspection helps accelerate production by up to $50 \%$ Lightweight Design worldwide

5. Meister S, Wermes MAM, Stueve J, Groves RM (2020) Algorithm assessment for layup defect segmentation from laser line scan sensor based image data. In: Zonta D, Huang H (eds) Sensors and Smart Structures Technologies for Civil, Mechanical, and Aerospace Systems, p 2020. SPIE. https://doi.org/10.1117/12. 2558434

6. European Machine Vision Association: Emva standard 1288 standard for characterization of image sensors and cameras. techreport 3.1, European Machine Vision Association (EMVA) (2016). https://www.emva.org/. Release 3.1

7. Chao J, Ward ES, Ober RJ (2016) Fisher information theory for parameter estimation in single molecule microscopy: tutorial. Journal of the Optical Society of America A 33(7):B36. https://doi.org/10.1364/josaa.33.000b36

8. Cemenska J, Rudberg T, Henscheid M (2015) Automated inprocess inspection system for AFP machines. SAE Int J Aerosp 8(2):303-309. https://doi.org/10.4271/2015-01-2608

9. Weimer C, Friedberger A, Helwig A, Heckner S, Buchmann C, Engel F (2016) Increasing the productivity of CFRP production processes by robustness and reliability enhancement. In: CAMX 2016 - The Composites and Advanced Materials Expo and Conference. Airbus Group Innovations, 81663 Munich, Germany; AirbusInfactory Solutions GmbH, 81663 Munich, Germany. https://www.researchgate.net/profile/ Christian_Weimer/publication/308778487_INCREASING_THE_ PRODUCTIVITY_OF_CFRP_PRODUCTION_PROCESSES_ BY_ROBUSTNESS_AND_RELIABILITY_ENHANCEMENT/ links/57efa78208ae886b8975147a.pdf

10. Black S (2018) Improving composites processing with automated inspection. compositesworld. https://www.compositesworld. com/articles/improving-composites-processing-with-automatedinspection. Https://www.compositesworld.com/articles/improvingcomposites-processing-with-automated-inspection

11. Ucan H, Scheller S, Nguyen DC, Nieberl D, Beumler T, Haschenburger A, Meister S, Kappel E, Prussak R, Deden D, Mayer M, Pantelelis N, Zapp P, Hauschild B, Menke N (2019) Automated, quality assured and high volume oriented production of fiber metal laminates (fml) for the next generation of passenger aircraft fuselage shells. In: The Fourth International Symposium on Automated Composites Manufacturing. https:// elib.dlr.de/127353/

12. Samajdar T, Quraishi MI (2015) Analysis and evaluation of image quality metrics. In: Advances in intelligent systems and computing, pp. 369-378. Springer india. https://doi.org/10.1007/978-81322-2247-7_38

13. Coyotl-Ocelotl B, Ramírez JCJ, Ramos-García R, Chiu R, Spezzia-Mazzocco T, Ramirez-San-Juan JC (2018) Speckle contrast calculation based on pixels correlation: spatial analysis. In: Morris MBN, Creath K, Burke J, Davies AD (eds) Interferometry XIX. SPIE. https://doi.org/10.1117/12.2321271

14. Wang F, Liu J, Liu Y, Wang Y (2016) Research on the fiber lay-up orientation detection of unidirectional CFRP laminates composite using thermal-wave radar imaging. NDT \&, E International 84:54-66. https://doi.org/10.1016/j.ndteint.2016.08.002

15. Lengsfeld H, Fabris FW, Krämer J, Lacalle J, Altstädt V (2014) Faserverbundwerkstoffe. Hanser Fachbuchverlag. https:// www.ebook.de/de/product/22746074/hauke_lengsfeld_felipe_ wolff_fabris_johannes_kraemer_javier_lacalle_volker_altstaedt_ faserverbundwerkstoffe.html

16. Maass D (2012) Automated dry fiber placement for aerospace composites. In: Composites manufacturing 2012. Danobat
17. Grohmann Y, Stoffers N, Kühn A, Mahrholz T (2016) Development of the direct roving placement technology (DRP). In: ECCM17 - 17th European Conference on Composite Materials. https://elib.dlr.de/107706/

18. Campbell F (2004) Manufacturing Processes for Advanced Composites. Elsevier Science \& Technology. https://www.ebook. de/de/product/6827737/manufacturing_processes_for_advanced_ composites.html

19. Rudberg T (2019) Webinar: Building AFP system to yield extreme availability. CompositesWorld, Video

20. Oromiehie E, Prusty BG, Compston P, Rajan G (2019) Automated fibre placement based composite structures: Review on the defects, impacts and inspections techniques. Compos Struct 110987:224. https://doi.org/10.1016/j.compstruct.2019.110987

21. Harik R, Saidy CJ, Williams S., Gurdal Z, Grimsley B (2018) Automated fiber placement defect identity cards: cause, anticipation, existence, significance, and progression. In: SAMPE 18. https://www.researchgate.net/publication/ 326464139_Automated_fiber_placement_defect_identity_cards_ cause_anticipation_existence_significance_and_progression

22. Potter K (2009) Understanding the origins of defects and variability in composites manufacture. ICCM International Conferences on Composite Materials. http://iccm-central.org/ Proceedings/ICCM17proceedings/Themes/Plenaries/P1.5

23. Atkinson GA, Thornton TJ, Peynado DI, Ernst JD (2018) Highprecision polarization measurements and analysis for machine vision applications. In: 2018 7Th european workshop on visual information processing (EUVIP). IEEE. https://doi.org/10.1109/ euvip. 2018.8611762

24. Schöberl M., Kasnakli K, Nowak A (2016) Measuring strand orientation in carbon fiber reinforced plastics (CFRP) with polarization. In: 19Th world conference on non-destructive testing 2016

25. Denkena B, Schmidt C, Völtzer K., Hocke T (2016) Thermographic online monitoring system for automated fiber placement processes. Composites Part B:, Engineering 97:239-243. https://doi.org/10.1016/j.compositesb.2016.04.076

26. Gregory ED, Juarez PD (2018) In-situ thermography of automated fiber placement parts. In: AIP Conference proceedings. https://doi.org/10.1063/1.5031551

27. Schmidt C, Hocke T, Denkena B (2019) Artificial intelligence for non-destructive testing of CFRP prepreg materials Production Engineering. https://doi.org/10.1007/s11740-019-00913-3

28. Gardiner G (2018) Zero-defect manufacturing of composite parts. CompositesWorld. https://www.compositesworld. $\mathrm{com} / \mathrm{blog} /$ post/zero-defect-manufacturing-of-composite-parts. Https://www.compositesworld.com/blog/post/ zero-defect-manufacturing-of-composite-parts

29. Black S (2018) Improving composites processing with automated inspection, part II. compositesworld. https://www.compositesworld. com/articles/improving-composites-processing-with-automatedinspection-part-ii. Https://www.compositesworld.com/articles/ improving-composites-processing-with-automated-inspection

30. Sun S, Han Z, Fu H, Jin H, Dhupia JS, Wang Y (2020) Defect characteristics and online detection techniques during manufacturing of FRPs using automated fiber placement: A review. Polymers 12(6):1337. https://doi.org/10.3390/polym12061337

31. Forest J, Salvi J, Cabruja E, Pous C (2004) Laser stripe peak detector for $3 \mathrm{~d}$ scanners. a FIR filter approach. In: Proceedings of the 17th International Conference on Pattern Recognition, 2004. ICPR 2004. IEEE. https://doi.org/10.1109/icpr.2004.1334612

32. Automation Technology GmbH: The FIR filter. techreport 1.0, Automation Technology GmbH, Hermann-Bössow-Straße 6-8, 23843 Bad Oldesloe, Germany (2014). https://www.stemmerimaging.com/media/uploads/cameras/12/122195-Automation_Technology_AppNote_FIR_Filter.pdf. Rev. 1.0 
33. Schmitt R, Niggemann C, Mersmann C (2008) Contour scanning of textile preforms using a light-section sensor for the automated manufacturing of fibre-reinforced plastics. In: Berghmans F, Mignani AG, Cutolo A, Meyrueis PP, Pearsall TP (eds) Optical Sensors 2008, vol. 7003, pp. 436-447. SPIE. https://doi.org/10.1117/12.779005

34. Schmitt R, Orth A, Niggemann C (2007) A method for edge detection of textile preforms using a light-section sensor for the automated manufacturing of fibre-reinforced plastics. In: Osten W, Gorecki C, Novak EL (eds) Optical Measurement Systems for Industrial Inspection V. SPIE. https://doi.org/10.1117/12.726177

35. Miesen N, Sinke J, Groves RM, Benedictus R (2015) Simulation and detection of flaws in pre-cured CFRP using laser displacement sensing. The International Journal of Advanced Manufacturing Technology 82(1-4):341-349. https://doi.org/10.1007/s00170015-7305-X

36. Amir YM, Thörnberg B (2017) High precision laser scanning of metallic surfaces. International Journal of Optics 2017:1-13. https://doi.org/10.1155/2017/4134205

37. Jähne B (2010) EMVA 1288 Standard for machine vision. Optik \&, Photonik 5(1):53-54. https://doi.org/10.1002/opph.201190082

38. Rosenberger M, Zhang C, Votyakov P, Preißler M, Notni G (2016) EMVA 1288 camera characterisation and the influences of radiometric camera characteristics on geometric measurements. ACTA IMEKO 5(4):81. https://doi.org/10.21014/acta-imeko.v5i4.356. https://www.researchgate.net/publication/312035490_EMVA_ 1288_Camera_characterisation_and_the_influences_of_ radiometric_camera_characteristics_on_geometric_measurements

39. Jähne B (2005) Digital Image Processing Springer-Verlag GmbH. https://doi.org/10.1007/3-540-27563-0

40. Jähne B (2012) Digitale bildverarbeitung springer berlin heidelberg. https://doi.org/10.1007/978-3-642-04952-1

41. Kube G (2016) Performance Studies of Industrial CCD Cameras Based on Signal-To-Noise and Photon Transfer Measurements. In: Proceedings of the 5th International Beam Instrumentation Conference. International Beam Instrumentation Conference, Barcelona (Spain), 11 Sep 2016 - 15 Sep 2016, JACoW, Geneve. https://doi.org/10.18429/JACoW-IBIC2016-TUPG76. http:// accelconf.web.cern.ch/ibic2016/doi/JACoW-IBIC2016-TUPG76. html

42. Jauregui-Sanchez $\mathrm{Y}$, Clemente $\mathrm{P}$, Latorre-Carmona $\mathrm{P}$, Tajahuerce E, Lancis J (2018) Signal-to-noise ratio of singlepixel cameras based on photodiodes. Appl Opt 57(7):B67. https://doi.org/10.1364/ao.57.000b67

43. Stokes-Griffin C, Compston P (2015) Optical characterisation and modelling for oblique near-infrared laser heating of carbon fibre reinforced thermoplastic composites. Opt Lasers Eng 72:1-11. https://doi.org/10.1016/j.optlaseng.2015.03.016

44. Zhao X, Shi X, Liu K, Deng Y (2019) An intelligent detection and assessment method based on textile fabric image feature. International Journal of Clothing Science and Technology 31(3):390-402. https://doi.org/10.1108/ijcst-01-2018-0005

45. Picotronic GmbH: Datasheet laser picotronic dc650-1-3(8x25)c500. techreport Rev. 2, Picotronic GmbH, Rudolf-Diesel-Str. 2a, 56070 Koblenz (2011). https://shop.picotronic.de/ Picotronic-DC650-1-3-8x25-C500/70114331

46. Automation Technology GmbH: C5 Series - User Manual for High Speed 3D Sensors. AT - Automation Technology GmbH, Hermann-Bössow-Straße 6-8, 23843 Bad Oldesloe, Germany, 1 edn. (2016). https://www.automationtechnology.de/cms/c5-serie/. Rev 1.0

47. Automation Technology GmbH: C5 series - user manual for high speed 3d sensors. techreport 1.2, Automation Technology GmbH, Hermann-Bössow-Straße 6-8, 23843 Bad Oldesloe, Germany (2019). https://www.automationtechnology.de/cms/wp-content/ uploads/2019/03/C5-Series_specifications_web.pdf. Rev 1.2

48. ams AG: Datasheet ds000603 - cmv12000 - cmos image sensor. techreport 3.0, ams AG, Tobelbader Strasse 30, 8141 Premstaetten, Austria (2020). https://ams.com/documents/20143/ 36005/CMV12000_DS000603_3-00.pdf/d27f4643-e11b-86f9-4e09ec055cb4c8e1. Datasheet DS000603 v3-00

49. Hexcel Corporation: Hexply 8552 - datasheet. techreport, Hexcel Corporation (2020). https://www.hexcel.com/user_area/content_ media/raw/HexPly_8552_eu_DataSheet.pdf

50. Hexcel Corporation: Hextow im 7 - carbon fiber. techreport, Hexcel Corporation (2020). https://www.hexcel.com/user_area/ content_media/raw/IM7_HexTow_DataSheet.pdf

51. Opsira GmbH: gonio'2pi. techreport, Opsira GmbH, Leibnizstraße 20, 88250 Weingarten, Germany (2019). https://www.opsira. de/fileadmin/benutzerdaten/opsira-de/pdf/Product_information near-field_goniophotometer_gonio_2pi_-_E_-_V00263114_-_ opsira.pdf

52. Jos. Schneider Optische Werke GmbH: Anti-shading lens - xenoplan 2.8/50-0902. techreport 2.0, Jos. Schneider Optische Werke GmbH (2008). https://schneiderkreuznach.com/application/files/ 3415/0839/4884/XNP-2.8-50-anti-shading.pdf. Vers. 2.0

53. Midwest Optical Systems: Bp660 dark red bandpass filter. techreport, Midwest Optical Systems, 322 Woodwork Lane, Palatine, IL 60067 USA (2020). https://midopt.com/filters/bp660/

54. Dainty JC (1975) Laser speckle and related phenomena. SpringerVerlag, Berlin New York

55. Thompson O, Andrews M, Hirst E (2011) Correction for spatial averaging in laser speckle contrast analysis. Biomedical Optics Express 2(4):1021. https://doi.org/10.1364/boe.2.001021

56. Tutsch R, Han S, Dierke H (2015) Speckle reduction for a laser light sectioning sensor. MATEC Web of Conferences 32:06005. https://doi.org/10.1051/matecconf/20153206005

57. Paul H (2003) Lexikon der Optik : in zwei bänden. spektrum, Akad. Verl Heidelberg

58. Sheikh H, Sabir M, Bovik A (2006) A statistical evaluation of recent full reference image quality assessment algorithms. IEEE Trans Image Process 15(11):3440-3451. https://doi.org/10.1109/ tip. 2006.881959

59. Welstead S (1999) Fractal and wavelet image compression techniques. SPIE Optical Engineering Press, Bellingham Wash

60. Barni M (2006) Document and image compression. CRC/taylor \& Francis, Boca Raton FL

61. Automation Technology GmbH: C5 Series - User Manual for High Speed 3D Sensors. AT - Automation Technology GmbH, Hermann-Bössow-Straße 6-8, 23843 Bad Oldesloe, Germany, 1.4 edn. (2019). https://www.automationtechnology.de/cms/c5-serie/. Rev 1.4

62. Kay, S.M.: Fundamentals of Statistical Processing, Volume I. Prentice Hall (1993). https://www.ebook.de/de/product/3640711/ steven_m_kay_fundamentals_of_statistical_processing_volume_i. html

Publisher's note Springer Nature remains neutral with regard to jurisdictional claims in published maps and institutional affiliations. 